"This is the peer reviewed version of the following article: Topics in Current Chemistry 2016, In press

DOI: 10.1007/s41061-016-0045-Z, which has been published in

final form at http://link.springer.com/article/10.1007/s41061-016-0045-

z/fulltext.html?wt_mc=alerts.TOCjournals]. This article may be used for non-commercial purposes

in accordance with Springer Terms and Conditions for Self-Archiving."

\title{
Ni- and Fe-catalyzed carboxylation of unsaturated hydrocarbons with $\mathrm{CO}_{2}$
}

Francisco Juliá-Hernández, ${ }^{1}$ Morgane Gaydou, ${ }^{1}$ Eloisa Serrano, ${ }^{1}$ Manuel van Gemmeren, ${ }^{1}$ Ruben Martin*1,2

${ }^{1}$ Institute of Chemical Research of Catalonia (ICIQ), The Barcelona Institute of Science and Technology, Av. Països Catalans 16, 43007 Tarragona, Spain

${ }^{2}$ Catalan Institution for Research and Advanced Studies (ICREA), Passeig Lluïs Companys, 23, 08010, Barcelona, Spain

*rmartinromo@iciq.es

\begin{abstract}
The sustainable utilization of available feedstock materials for preparing valuable compounds holds great promise to revolutionize approaches in organic synthesis. In this regard, the implementation of abundant and inexpensive carbon dioxide $\left(\mathrm{CO}_{2}\right)$ as a $\mathrm{C} 1$ building block has recently attracted a considerable attention. Among the different alternatives in $\mathrm{CO}_{2}$ fixation, the preparation of carboxylic acids, relevant motifs in pharmaceuticals and agrochemicals, is particularly appealing, thus providing a rapid and unconventional entry to building blocks that are typically prepared via wasteproducing protocols. While significant advances have been realized, the utilization of simple unsaturated hydrocarbons as coupling partners in carboxylation events is undoubtedly of utmost academic and industrial relevance, as two available feedstock materials can be combined in a catalytic fashion. This review article aims to describe the main achievements on the direct carboxylation of unsaturated hydrocarbons with $\mathrm{CO}_{2}$ by using cheap and available Ni or Fe catalytic species.
\end{abstract}

Keywords Nickel $\cdot$ Iron $\cdot \mathrm{CO}_{2} \cdot$ carboxylation $\cdot$ unsaturated hydrocarbons $\cdot$ catalysis $\cdot$ carboxylic acids $\cdot$ cross-coupling 


\section{Introduction}

The atmospheric concentration of carbon dioxide $\left(\mathrm{CO}_{2}\right)$ has been dramatically raised in the last decades as result of the industrial development of our society. The ever-growing concentration of $\mathrm{CO}_{2}$ has led to discussions on how to alleviate the effects of the climatic change [1]. However, $\mathrm{CO}_{2}$ is an abundant, inexpensive and renewable feedstock that could be potentially used as a $\mathrm{C} 1$ building block for synthesis [2-4]. Although the implementation of $\mathrm{CO}_{2}$ fixation in synthetic methods will certainly not reduce its concentration in the atmosphere, it could be transformed into high value-added fine chemicals. In fact, more than 110 megatons of $\mathrm{CO}_{2}$ are annually used in industry for the synthesis of urea, salicylic acid and carbonates [1,5]. However, $\mathrm{CO}_{2}$ is a highly oxidized and thermodynamically stable gas and, consequently, high activation energies are required for its functionalization [6-10].

Carboxylic acids rank amongst the most prevalent backbones in pharmaceuticals and agrochemicals. The main strategies for the synthesis of carboxylic acids are based on the oxidation of primary alcohols and aldehydes, and the hydrolysis of nitriles [11]. However, these methods suffer from the use of harsh conditions, strong oxidants and/or high temperatures, inevitably resulting in a poor chemoselectivity profile. Alternatively, chemists have designed new routes for preparing carboxylic acids via direct carboxylation of well-defined organometallic species in which $\mathrm{CO}_{2}$ inserts into a highly energetic metal-carbon bond that forms the corresponding carboxylic acid upon hydrolytic work-up [12]. While a significant step-forward, such a method is not particularly step-economical and requires handling with stoichiometric and, in many instances, air-sensitive organometallic species, thus reinforcing a change in strategy. Although the recent years have witnessed the discovery of a myriad of elegant catalytic reductive carboxylation techniques with organic (pseudo)halides [13-16], the utilization of unsaturated hydrocarbon counterparts constitutes an ideal platform in the carboxylation arena, as these motifs can be obtained in bulk from our petrochemical industry, thus representing a formidable and unique opportunity for converting raw materials into valuable products at industrial level [17]. Additionally, such a scenario would allow for accessing carboxylic acids at large scale while avoiding the utilization of toxic carbon monoxide [11].

The direct carboxylation of organic compounds has traditionally been associated with the utilization of Ni complexes, an observation that can be traced back from the seminal work of Aresta and coworkers by the isolation of the first $\mathrm{CO}_{2}$ complex to a transition metal $\left[\mathrm{Ni}\left(\eta^{2}-\mathrm{CO}_{2}\right)\left(\mathrm{PCy}_{3}\right)_{2}\right]$ [18]. In the last three decades, $\mathrm{CO}_{2}$ fixation into unsaturated hydrocarbons has garnered considerable attention from the scientific community, either using stoichiometric or catalytic amounts of transition metals, reaching remarkable levels of sophistication, efficiency and applicability. Given the preparative potential of these transformations, we identified the need to review the most prominent advances in this field of expertise. Unlike other reviews for similar means, the purpose of this article is to focus on the most recent advances for preparing carboxylic acid derivatives from unsaturated hydrocarbon 
backbones using $\mathrm{Ni}$ as well as the cheaper and more abundant Fe species in a homogeneous manner, including mechanistic considerations, when appropriate. Therefore, other catalytic $\mathrm{CO}_{2}$ functionalization processes of paramount importance such as the area of cyclic carbonates [19-21], polymer formation, production of methanol or formic acid [22-24], electrochemical methods [25], Cocatalyzed carboxylations [26], carbonylation methods [27] or reductive carboxylations of organic (pseudo)halides [14-16; 28-29], among others, will be beyond the scope of this review.

\section{Carboxylation of alkynes}

\subsection{Stoichiometric processes}

Prompted by the seminal Ni-catalyzed oxidative cyclization work of Inoue for preparing 2-pyrones using $\mathrm{CO}_{2}$ as coupling partner [30], Burkhart and Hoberg reported the isolation of an oxanickelacyclopentene (nickelalactone) from the coupling of 2-butyne and $\mathrm{CO}_{2}$ with $\mathrm{Ni}(\mathrm{cdt}$ ) (cdt = 1,5,6-cyclododecatriene) and $N, N, N^{\prime}, N^{\prime}$-tetramethylethylenediamine (TMEDA) [31]. The authors highlighted that the corresponding $\alpha, \beta$-unsaturated carboxylic acid can be easily within reach upon simple protonolysis (Scheme 1).

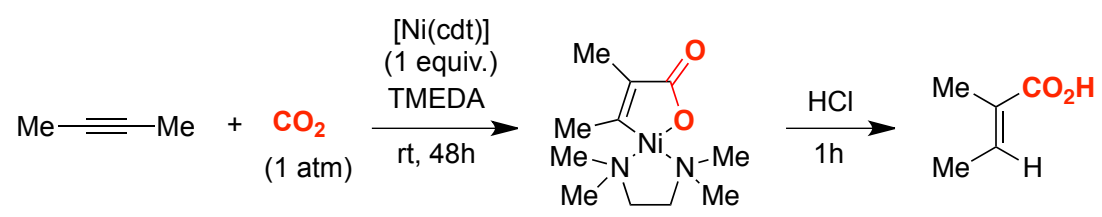

Scheme 1. Synthesis of acrylic acids via the intermediacy of nickelalactones [31]

The intermediacy of nickelalactones was further corroborated by theoretical calculations [32-35]. Specifically, in 2008 Buntine and co-workers modelled the reaction with acetylene and $\mathrm{CO}_{2}$ [34], selecting $m \mathrm{DBU}$ as a model ligand to mimic the behavior of DBU (DBU = diazabicyclo[5.4.0]undec7 -ene). The authors found that the reaction proceeds through an associative mechanism, first involving the $\eta^{2}$-coordination of the alkyne to the nickel(0) complex followed by a direct insertion of $\mathrm{CO}_{2}$ (Scheme 2). The reaction turned out to be thermodynamically favored, with an activation barrier of $23.9 \mathrm{kcal} / \mathrm{mol}$ for the key oxidative cyclization event without going through an Aresta-type complex.

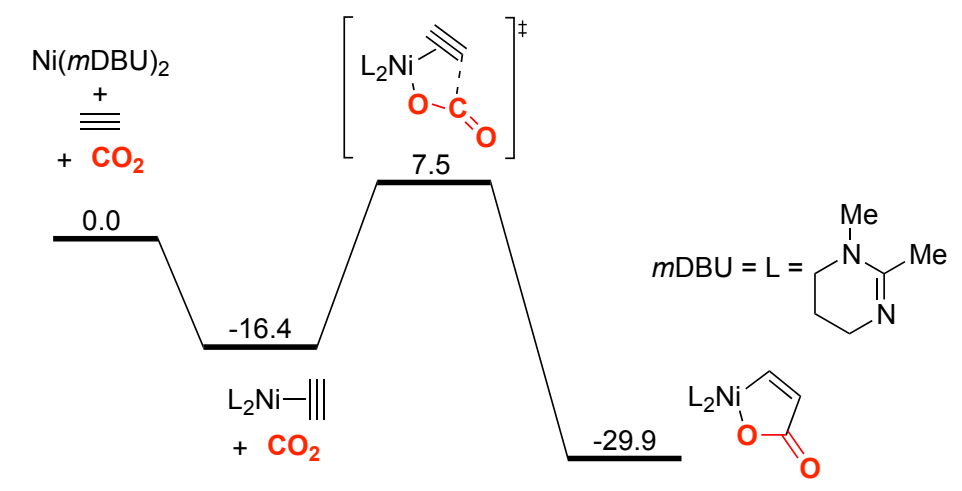

Scheme 2. Gibbs free energies (kcal/mol) for the coupling of $\mathrm{CO}_{2}$ with acetylene [34] 
Strikingly, it took more than 15 years until Yamamoto and Saito reported the preparation of nickelalactones with terminal alkynes [36]. In this work, the authors disclosed the stoichiometric carboxylation of terminal alkynes with in situ formed $\mathrm{Ni}(\mathrm{DBU})_{2}$, leading to the rapid formation of $\beta$ substituted carboxylic acids with high levels of chemo- and regioselectivity (Scheme 3). This reaction could be applied to differently substituted alkynes bearing aromatic, aliphatic or silyl groups with equal ease.
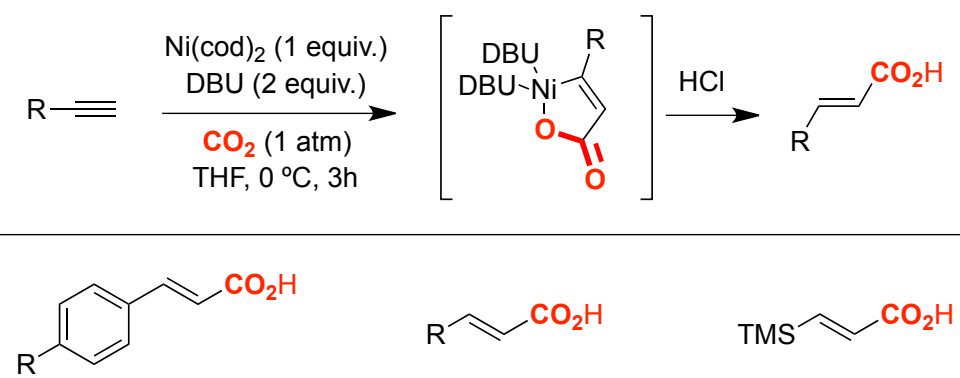

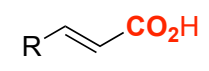

$\mathrm{R}=\mathrm{H}, 85 \%$

$\mathrm{R}=\mathrm{OMe}, 86 \%$

$\mathrm{R}=\mathrm{CF}_{3}, 91 \%$
$\mathrm{R}=t \mathrm{Bu}, 72 \%$

$\mathrm{R}=n \mathrm{Hex}, 74 \%(16: 1)$<smiles>CC=CC(=O)O</smiles>

$58 \%$

Scheme 3. Nickel(0)-mediated carboxylation of terminal alkynes by Yamamoto and Saito [36]

The regioselectivity observed in the oxidative cyclization of monosubstituted alkynes with $\mathrm{CO}_{2}$ was theoretically rationalized by Buntine (Scheme 4) [34]. The authors observed a markedly different energetic preference for the two possible transition states (TS-I and TS-II), with a lower energy barrier for TS-I leading to $\mathbf{I}$, in which $\mathrm{CO}_{2}$ insertion takes place distal to the substituent on the alkyne motif. However, nickelalactone II turned out to be thermodynamically favored due to the avoidance of a steric clash of the substituent on the alkyne terminus with the Ni center. The effect of the ancillary ligand was also investigated, with DBU leading to lower activation energies when compared to 2,2'bipyridine (bpy). It was particularly interesting to find out that polar solvents such as DMF lowered down all activation barriers due to the stabilizing effect of DMF on the transition states when compared with commonly employed tetrahydrofuran (THF).

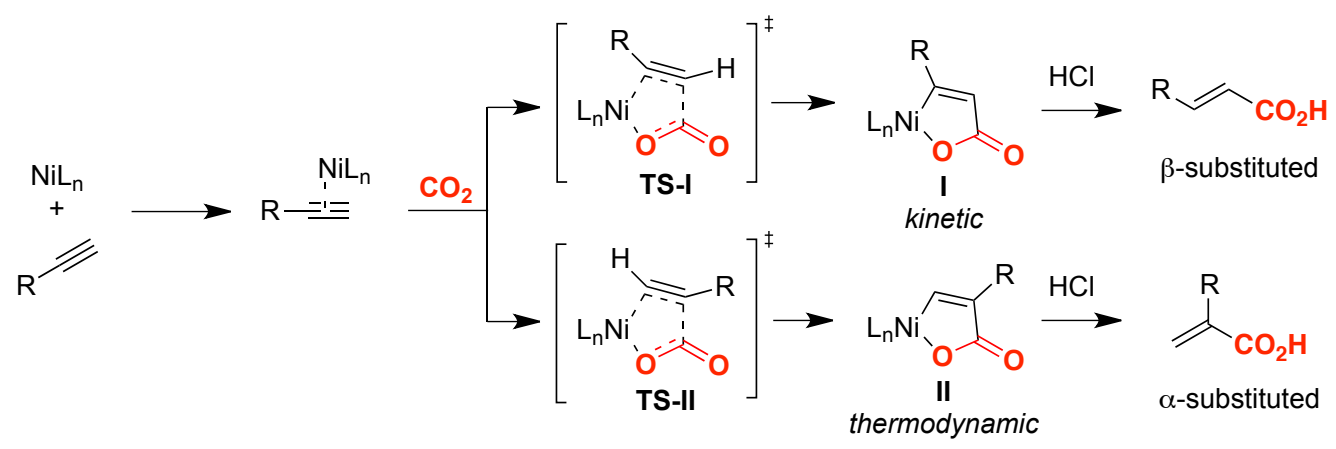

Scheme 4. Regioselectivity profile in the cycloaddition of terminal alkynes with $\mathrm{CO}_{2}[34]$

In 2004, the group of Iwasawa reported a similar study for the stoichiometric carboxylation of terminal and unsymmetrical alkynes using a series of bis(amidine)ligands [37]. Interestingly, the 
nature of the ligand dictated the regioselectivity pattern; while ligands bearing substituents at the methylene carbon generated predominantly cinnamic acids in good yields, less substituted bis(amidine) ligands resulted in a switch of selectivity, leading preferentially to $\alpha$-substituted carboxylic acids, albeit in lower yields (Scheme 5). Although this transformation still required stoichiometric amounts of nickel, this work represented a formidable step forward for promoting a ligand-controlled regiodivergent carboxylation of alkynes.

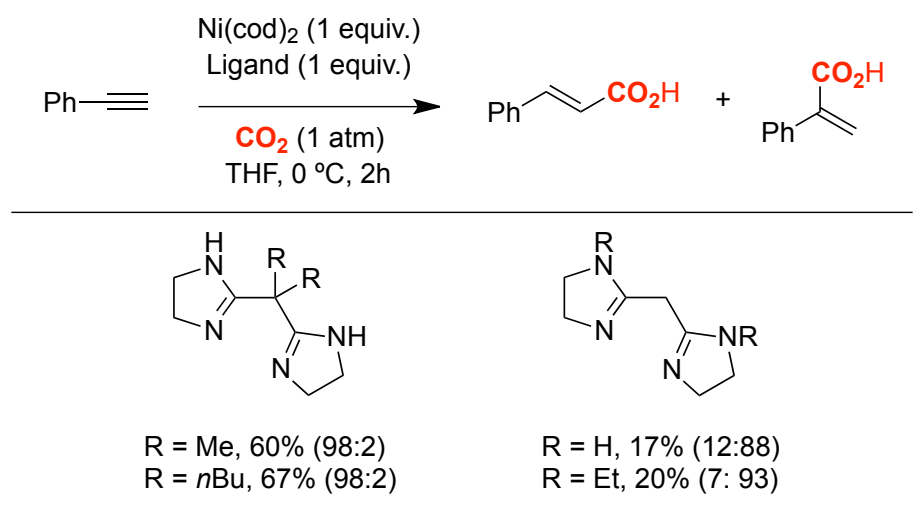

Scheme 5. Bis(amidine)ligands in Ni-mediated synthesis of acrylic acids [37]

Mori and co-workers significantly extended the application profile of the Ni-mediated carboxylation of alkynes with $\mathrm{CO}_{2}$ by using organozinc reagents as coupling partners, thus triggering a transmetalation with the in situ generated nickelalactone, and giving rise to a formal alkylative or arylative carboxylation of terminal alkynes depending on the organozinc reagent utilized [38]. The reaction proceeded under mild conditions and a wide range of organozinc reagents could be used leading to $\beta, \beta$ '-disubstituted unsaturated carboxylic acids with an excellent regioselectivity profile that goes in line with the hydrocarboxylation procedure reported by Yamamoto and Saito (Scheme 6). [36]. Notably, the authors found that heterocyclic structures were within reach by appropriately locating a tethered heteroatom on the side-chain, thus setting the stage for an intramolecular Michael addition $[39,40]$.

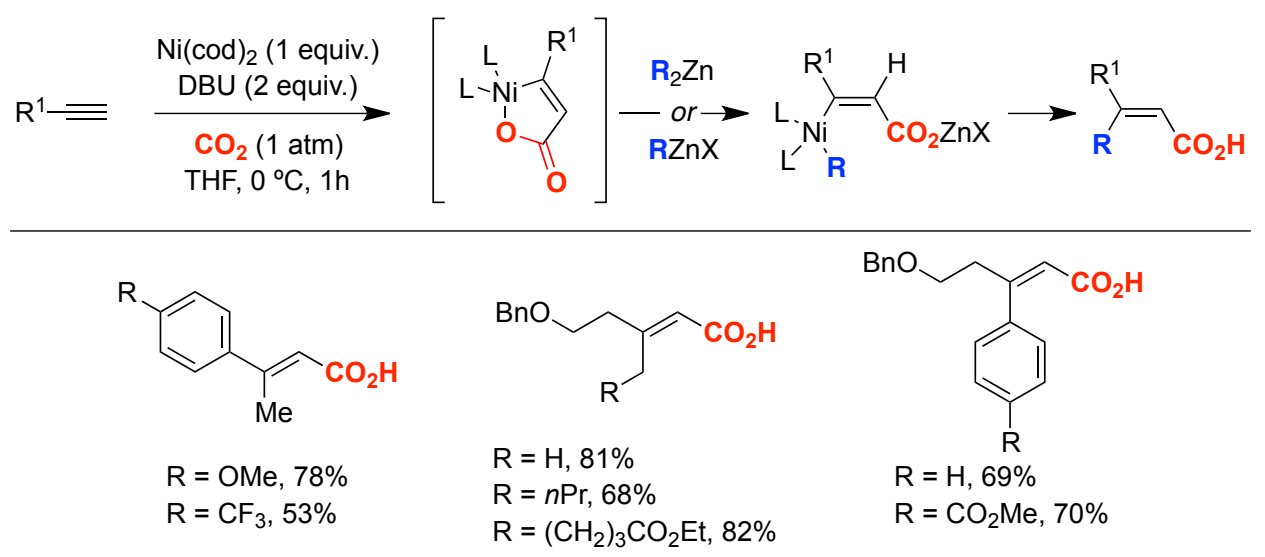

Scheme 6. Ni(0)-mediated alkylative/arylative carboxylation of alkynes [38] 
The nickel(0)-promoted carboxylation of alkoxy acetylenes has been recently developed by the group of Sato and Saito [41]. The authors predicted that the electron-donating properties of an ether substituent could dictate the regioselectivity profile when forming the intermediate nickelalactone, thus leading to $\mathrm{CO}_{2}$ insertion onto the most nucleophilic carbon. As shown in Scheme 7, this turned out to be the case. Such an outcome is noteworthy as an opposite selectivity pattern was observed by Yamamoto and Saito, in which $\mathrm{CO}_{2}$ insertion occurred at the less sterically congested carbon. The applicability of this transformation was further corroborated by a subsequent Rh-catalyzed asymmetric hydrogenation, affording enantioenriched $\beta$-aryloxypropionic acid derivatives.

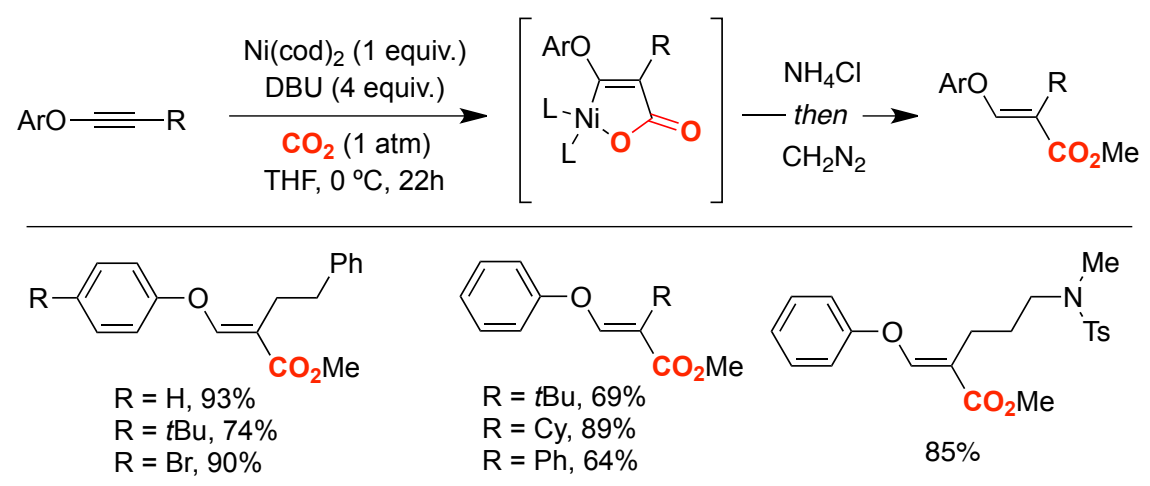

Scheme 7. Ni(0)-promoted regioselective carboxylation of alkoxy acetylenes [41]

\subsection{Catalytic carboxylation of alkynes}

The first metal-catalyzed carboxylation of alkynes with $\mathrm{CO}_{2}$ was reported three years before the isolation of nickelalactones by Hoberg [31]. Specifically, in 1977 Inoue described the carboxylation of alkynes with $\mathrm{CO}_{2}$ leading to the formation of 2-pyrones. The utilization of terminal acetylenes such as 1-hexyne in combination with a bidentate phosphine afforded the corresponding 2-pyrone in low yields, together with inevitable trimerization side products. (Scheme 8) [30]. As expected, the parasitic trimerization could be avoided when using internal alkynes, affording high yields of the tetrasubstituted-2-pyrones [42,43]. Further improvements on reactivity and selectivity were achieved by Walther [44] and Saegusa [45,46], revealing a non-negligible effect on the nature of the ligand utilized in order to obtain the targeted 2-pyrones with high yields and selectivities.

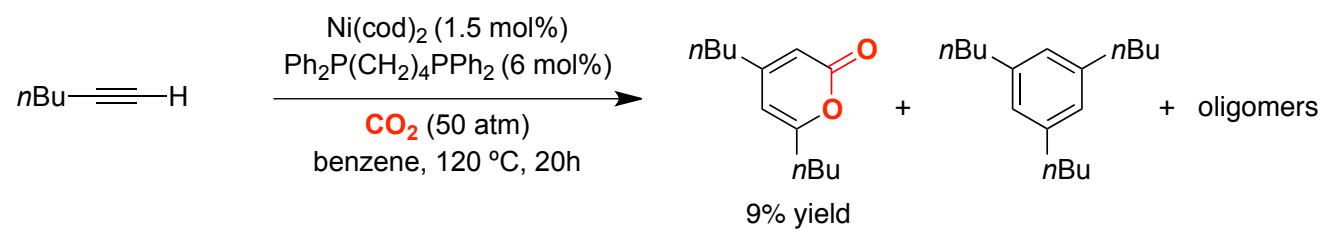

Scheme 8. First $\mathrm{Ni}(0)$-catalyzed cycloaddition of alkynes with $\mathrm{CO}_{2}$ en route to 2-pyrones [30]

Inoue tentatively proposed a mechanism based on the intermediacy of a nickelacyclopentadiene formed via oxidative cyclization of two alkyne moieties. Subsequent $\mathrm{CO}_{2}$ insertion into the $\mathrm{Ni}-\mathrm{C}$ bond or an alternative [4+2] cycloaddition were proposed as conceivable pathways towards the 
corresponding 2-pyrones. Few years later, Hoberg's stoichiometric studies unambiguously revealed that this reaction proceeds via oxidative cyclization of an alkyne, $\mathrm{CO}_{2}$ and $\mathrm{Ni}(0)$, thus forming the corresponding nickelalactone that would ultimately insert a second alkyne molecule to afford 2pyrones upon final reductive elimination [45-47]. A comprehensive review on the use of the oxidative cyclization techniques starting from alkynes and $\mathrm{CO}_{2}$ was nicely reported by Mori [48].

\subsubsection{Reductive carboxylation of alkynes}

Prompted by their previous work on the Ni-mediated carboxylation of disubstituted alkynes [38] (Scheme 6), Mori reported the Ni-catalyzed synthesis of $\alpha$-silyl- $\beta, \beta$ '-dialkyl $\alpha, \beta$-unsaturated carboxylic acids from silyl-substituted alkynes in presence of zinc reagents and an excess of DBU (Scheme 9). Although the reaction affords tetrasubstituted alkenes with a high regioselectivity and good yields, the methodology was unfortunately limited to $\mathrm{Me}_{2} \mathrm{Zn}, \mathrm{Bu}_{2} \mathrm{Zn}, \mathrm{Ph}_{2} \mathrm{Zn}$ or $\mathrm{Bn}_{2} \mathrm{Zn}$ as reagents $[49,50]$. The regioselectivity was explained by the preferential formation of $\mathbf{A}$ in which the Ni center is located distal to the silyl group, an issue that can be interpreted on the basis of both electronic as well as steric effects [51]. Such finding was further corroborated in subsequent theoretical calculations in which $\mathbf{A}$ was favored both from a kinetic and thermodynamic standpoint [34]. Not surprisingly, a selectivity switch was observed for alkynes not possessing a silyl group, such as tert-butyl or aromatic motifs. The synthetic utility of this methodology was showcased on a short total synthesis of Tamoxifen, an antiestrogenic anticancer drug, in 36\% overall yield from $p$-iodophenol, using a Nicatalyzed carboxylation of a phenyl-substituted acetylene with $\mathrm{CO}_{2}$ and $\mathrm{Ph}_{2} \mathrm{Zn}$ as the key step [50].

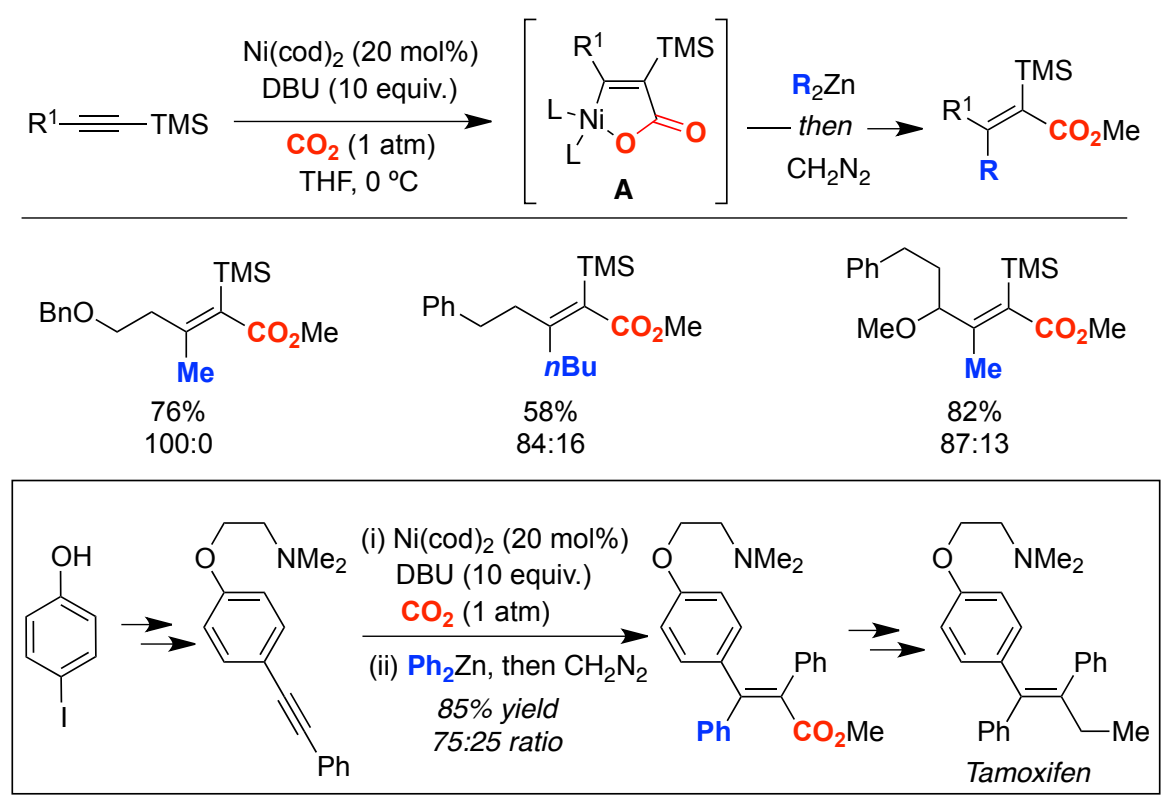

Scheme 9. Ni-catalyzed carboxylation of silyl-substituted alkynes with $R_{2} Z n[49,50]$.

The double carboxylation of alkynes to afford maleic anhydrides using Ni precatalysts, $\mathrm{Zn}$ as reducing agent and $\mathrm{MgBr}_{2}$ as additive, has recently been developed by Tsuji and Fujihara (Scheme 10) [52]. Based on stoichiometric and DFT studies, the authors proposed a catalytic cycle consisting of the 
initial formation of Hoberg's nickelalactone, followed by one-electron reduction with $\mathrm{Zn}$ in the presence of $\mathrm{MgBr}_{2}$ to generate $\mathbf{B}$. A second $\mathrm{CO}_{2}$ insertion into the Ni-C bond is mediated by the coordination of a $\mathrm{MgBr}^{+}$fragment to both $\mathrm{CO}_{2}$ and the carboxylate moiety. Finally, one-electron reduction by $\mathrm{Zn}$ affords the dicarboxylated species that ultimately are converted into the corresponding maleic anhydride while recovering back the active propagating $\mathrm{Ni}(0)$ species.

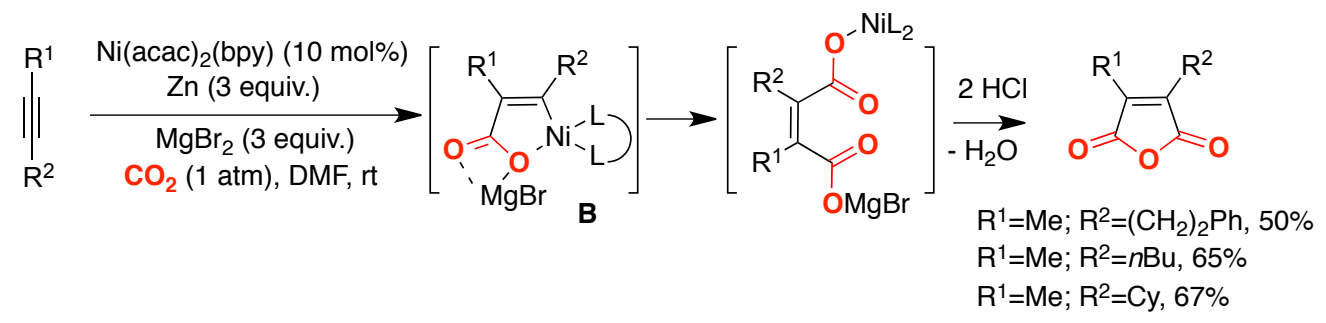

Scheme 10. Nickel-catalyzed double carboxylation of alkynes [52]

\subsubsection{Catalytic hydrocarboxylation of alkynes}

In 2011, the group of Ma described a nickel-catalyzed hydrocarboxylation of alkynes using $\mathrm{Ni}(\mathrm{cod})_{2}$ and diethylzinc as reducing agent (Scheme 11) [53]. The reaction turned out to be highly regio- and stereoselective, thus accessing syn-hydrocarboxylated products at 1-3 mol\% catalyst loadings. Although they employed stoichiometric amounts of cesium fluoride (CsF), no additional ligand was required, no doubt a formidable bonus when compared with other carboxylation techniques. While the mechanism remains speculative, the authors favored a transmetalation/ $\beta$-hydride elimination pathway, resulting in an alkenyl zinc derivative that ultimately reacts with $\mathrm{CO}_{2}$ to deliver the targeted carboxylic acid. Notably, $\mathrm{CO}_{2}$ insertion typically occurs adjacent to the aromatic site in unsymmetrically substituted alkynes or in close proximity to a directing group such as $N$-tosyl motifs. It is worth noting that an otherwise related work was independently described by Tsuji using $\mathrm{Cu}$ catalysts in combination with organosilanes as reducing agents [54]. Later on, Ma and co-workers reported an otherwise related hydrocarboxylation and methyl-carboxylation technique of homopropargylic alcohols in which the pending alcohol directs the carboxylation en route to $\alpha$-alkylidene- $\gamma$ butyrolactones $[55,56]$.

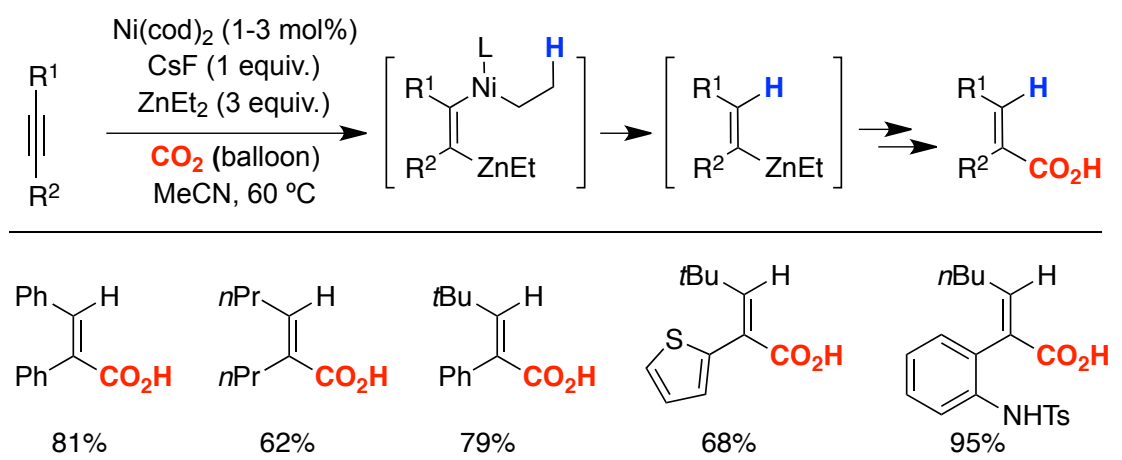

Scheme 11. Ni(0)-catalyzed syn-hydrocarboxylation of alkynes promoted by $\mathrm{Et}_{2} \mathrm{Zn}$ [53] 
More recently, Martin and co-workers described a novel Ni-catalyzed regioselective hydrocarboxylation of alkynes that obviates the need for stoichiometric and air-sensitive organometallic species by using simple alcohols as proton sources (Scheme 12) [57]. Importantly, such a transformation was distinguished by an intriguing regioselectivity profile in which $\mathrm{CO}_{2}$ insertion took place exclusively distal to the aromatic site, regardless of whether a directing group was present or not, and independently on the substitution pattern on the alkyne terminus, an observation that demonstrates the complementarity of this method when compared with other protocols using metal hydrides [58]. The origin of the regioselectivity profile was attributed to the intermediacy of two nickelalactones that were in equilibrium upon $\mathrm{CO}_{2}$ extrusion followed by a preferential binding of the alcohol motif to the $\mathrm{Ni}$ (II) center in $\mathbf{C}$, thus avoiding the clash with the bulkier aliphatic backbone. A subsequent two-electron reduction mediated by $\mathrm{Mn}$ recovers back the $\mathrm{Ni}(0) \mathrm{L}_{\mathrm{n}}$ species while delivering a manganese carboxylate that upon hydrolytic workup results in the expected acrylic acid. Notably, the mild reaction conditions as well as the absence of highly nucleophilic organometallic reagents allowed for an excellent chemoselectivity profile, as functional groups such as nitriles, amides, aldehydes or alkenes, among others, were perfectly tolerated.

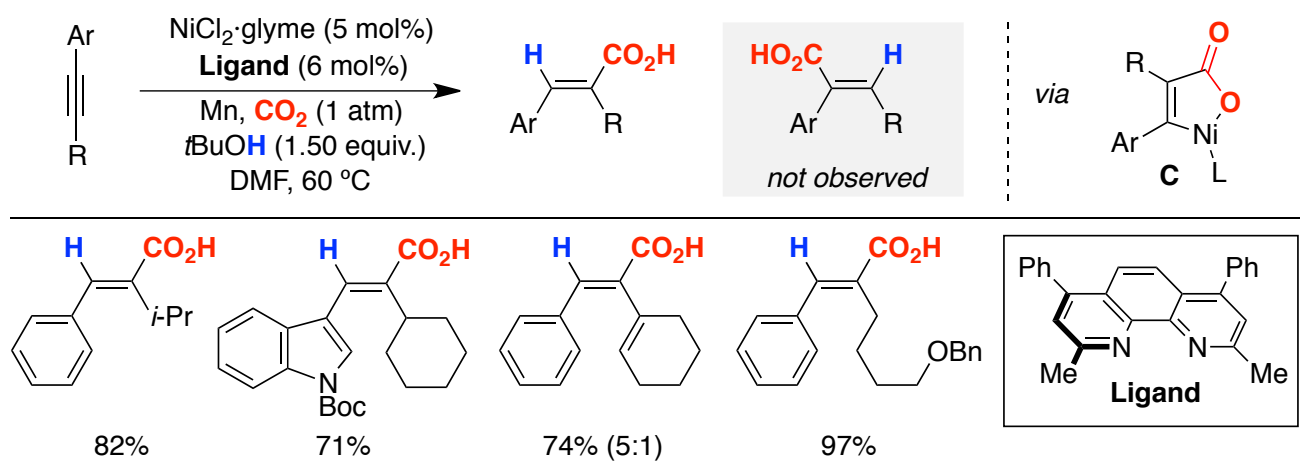

Scheme 12. Ni-catalyzed hydrocarboxylation of alkynes with alcohols as proton sources [57]

Although beyond the scope of this review, it is worth mentioning that a recent report by Fu has demonstrated that a Ni-catalyzed hydrocarboxylation of alkynes can be conducted using formic acid and a catalytic amount of anhydride via the in situ generation of carbon monoxide [59]. Likewise, a remarkable Co-catalyzed carboxyzincation of alkynes has recently been reported by Tsuji and Fujihara, allowing for preparing highly functionalized acrylic acids, even in a multicomponent fashion, and under mild reaction conditions [26].

\section{Carboxylation of alkenes}

\subsection{Stoichiometric processes involving alkenes}

In 1982, pioneering studies by Hoberg et al. showed that electron-rich $\mathrm{Ni}(0)$ species were able to promote the oxidative cyclization of olefins and $\mathrm{CO}_{2}$ in an analogous manner to that shown previously for alkyne backbones $[60,61]$. These processes gave rise to nickelalactones that were isolated and characterized with ethylene in the presence of bipyridine or bisphosphine ligands (Scheme 13) [61]. 
Protonolysis of these complexes led to the formation of the corresponding propionic acid in good yields, thus constituting a direct method to carboxylate alkenes in the presence of nickel complexes.

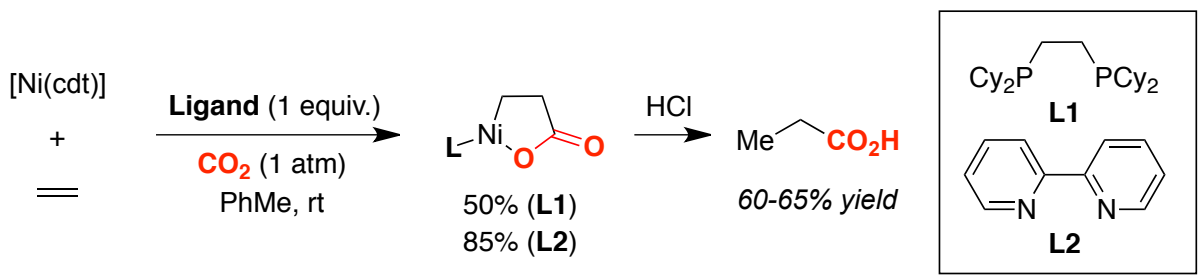

Scheme 13. Ni-mediated oxidative cyclization of ethylene and $\mathrm{CO}_{2}$ en route to propionic acid [61].

Prompted by this seminal work, efforts were subsequently focused on unravelling the fundamental features of the Ni-mediated oxidative cyclization of alkenes and $\mathrm{CO}_{2}$ in terms of regioselectivity and reaction mechanism. The regioselectivity of the corresponding 5 -membered metallacycles depends on the nature of the ligand, alkene and temperature. As shown in Scheme 13, both bipyridine and bisphosphine ligands can be equally used to prepare the corresponding nickelalactone. However, the formation of the 5-membered metallacycle with mono- and 1,2-disubstituted alkenes is more efficient with electron-rich imines DBU [62] and L1 [61]. Attempts to prepare a nickelalactone with aliphatic monosubstituted alkenes and $\mathrm{Ni} / \mathbf{L} 2$ systems resulted in the disproportionation of $\mathrm{CO}_{2}$ while forming $\mathrm{CO}$ and $\left[\mathrm{Ni}(\mathrm{bpy}) \mathrm{CO}_{3}\right][61]$. Interestingly, the electronic and steric nature of the alkene strongly determines product distribution in the cyclization process. Specifically, nickelalactones derived from styrene derivatives prefer to adopt a configuration in which the aryl substituent is located adjacent to the $\mathrm{Ni}$ center, due to a better stabilization of the $\mathrm{Ni}-\mathrm{C}$ bond (Scheme 14, equation a). In this manner, the corresponding linear carboxylic acid is obtained as the major product after a final protonolysis event. In contrast, the regioselectivity with aliphatic $\alpha$-olefins (monosubstituted alkenes) is dictated by steric effects, and the most stable metallacycle contains the Ni center coordinated to a primary carbon atom (Scheme 14, equation b). Accordingly, the corresponding $\alpha$-branched carboxylic acid is formed predominantly after protonolysis. It is worth mentioning that the regioselectivity in the oxidative cyclization with olefins differs from that shown with alkyne counterparts (Scheme 3). Interestingly, the reaction temperature heavily influenced the product distribution in the presence of aliphatic substituents. While a 4:1 ratio favoring the $\alpha$-branched carboxylic acid was found at room temperature, a negligible amount of linear carboxylic acid was observed at higher temperatures (25:1 ratio). This observation suggested that the two possible nickelalactones likely coexist in equilibrium upon $\mathrm{CO}_{2}$ extrusion when using bidentate phosphine ligands, an observation that was univocally corroborated with further experimentation with olefin crossover experiments [47]. 


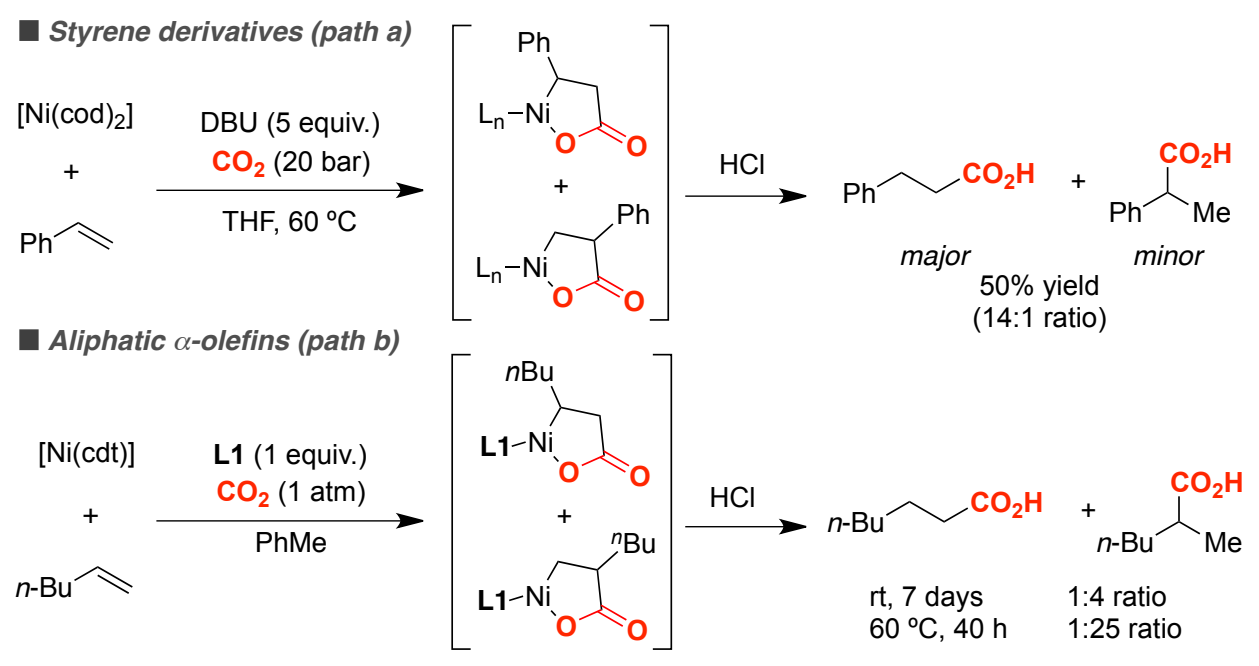

Scheme 14. Regioselectivity in the oxidative cyclization of alkenes and $\mathrm{CO}_{2}[61,62]$

The design of new ligands led to a further improvement of the regioselectivity in the cyclization with aliphatic olefins. Particularly, pyridyl-phosphine ligands (P,N ligands) with different electronic and steric properties afforded a strict control of the position of the substituents in the nickelalactone [63,64]. These ligands possess a push-pull character consisting of a mixture of electron-donating phosphine moieties with $\pi$-acidic pyridine fragments, which strongly stabilize the formation of the metallacycle, thus making the oxidative cyclization step irreversible. More recently, the formation of nickelalactones and subsequent protonolysis to give the corresponding saturated carboxylic acids could be achieved with activated trisubstituted olefins $[65,66]$. In this case, methylenecyclopropanes led to the corresponding cyclopropanecarboxylic acids in the presence of stoichiometric amounts of $\mathrm{Ni}(0)$ species. The selectivity towards the formation of the carboxylic acid respect to the propene ringopened product was dictated by the nature of ligand and solvent utilized.

Surprisingly, scarce mechanistic studies have been reported on the coupling of alkenes and $\mathrm{CO}_{2}$ in the presence of $\mathrm{Ni}(0)$ species. Experimentally, a pathway consisting of an oxidative cyclization from an Aresta-type $\mathrm{CO}_{2}$-coordinated $\mathrm{Ni}(0)$ complex [18] and an external alkene was ruled out, as the substitution of $\mathrm{CO}_{2}$ by the olefin was rapidly observed $[67,68]$. This was also corroborated by the fact that nickelalactones decomposed at high temperatures to give the corresponding Ni(0)-olefin complexes by extrusion of $\mathrm{CO}_{2}$ [47]. Interestingly, a more recent computational study shed light on the mode of operation of the oxidative cyclization reaction of ethylene and $\mathrm{CO}_{2}$ with both $\mathrm{Ni} / \mathbf{L} \mathbf{1}$ and $\mathrm{Ni} / \mathbf{L} 2$ systems [69]. Two fundamental aspects were explored: 1) the possible intermediacy of a $\mathrm{Ni}(0)-$ $\mathrm{CO}_{2}$ complex in the oxidative coupling process and 2) the nature of the transition state in the $\mathrm{C}-\mathrm{C}$ bond forming step. At the given level of theory, the DFT model could validate the experimental observation that in the Aresta-type complex, the $\mathrm{CO}_{2}$ ligand is substituted by ethylene in a process energetically favored by $1.4 \mathrm{kcal} / \mathrm{mol}$ with a negligible activation barrier. Therefore, an Aresta-type complex is not likely to be an intermediate in the oxidative coupling process. Additionally, Papai found that Hoberg's suggested 18-electron intermediate $\left[\mathrm{Ni}(\mathbf{L 2})\left(\mathrm{C}_{2} \mathrm{H}_{4}\right)\left(\mathrm{CO}_{2}\right)\right][47,61]$ should be rather 
unstable as it would exothermically dissociate $\mathrm{CO}_{2}$ with a negligible activation barrier of $0.8 \mathrm{kcal} / \mathrm{mol}$. As in the case of alkynes, this indicates that the $\mathrm{C}-\mathrm{C}$ bond forming step does not involve the simultaneous coordination of the unsaturated hydrocarbon and $\mathrm{CO}_{2}$ to $\mathrm{Ni}(0)$. Furthermore, although the optimization of a transition state was not possible, calculations delivered a local maximum stationary point via the analysis of the intrinsic reaction coordinate (IRC). This revealed that the formation of the $\mathrm{C}-\mathrm{C}$ bond would likely involve a single step in which the nickelalactone is formed from the ethylene-coordinated intermediate with an incoming molecule of $\mathrm{CO}_{2}$ (Scheme 15, a). Therefore, it seems that the simultaneous coordination of both $\mathrm{CO}_{2}$ and $\mathrm{C}_{2} \mathrm{H}_{4}$ is not indispensable for the formation of the nickelalactone. Calculations pointed towards a similar reaction pathway for $\mathbf{L} \mathbf{1}$ and other modified bipyridines and bidentante phosphines, even though with different energetic profiles. Further theoretical studies were carried with other different ligands, leading to similar conclusions [70]. Interestingly, in 2014, Limbach et al. theoretically proposed two different modes of addition of $\mathrm{CO}_{2}$ into the $\mathrm{Ni}(0)$ ethylene intermediates with bidentate phosphine ligands, consisting of an inner sphere and outer sphere mechanism (Scheme 15, b) [71]. In the former, the insertion is believed to occur in a single concerted step, as previously reported by the group of Papai. However, the direct attack of $\mathrm{CO}_{2}$ to the coordinated ethylene without earlier interaction with the Ni center (outer sphere pathway) was suggested to be energetically more viable in polar solvents (THF) and with bulky ligands such as 1,2-bis(ditert-butylphosphino)ethane (L3).
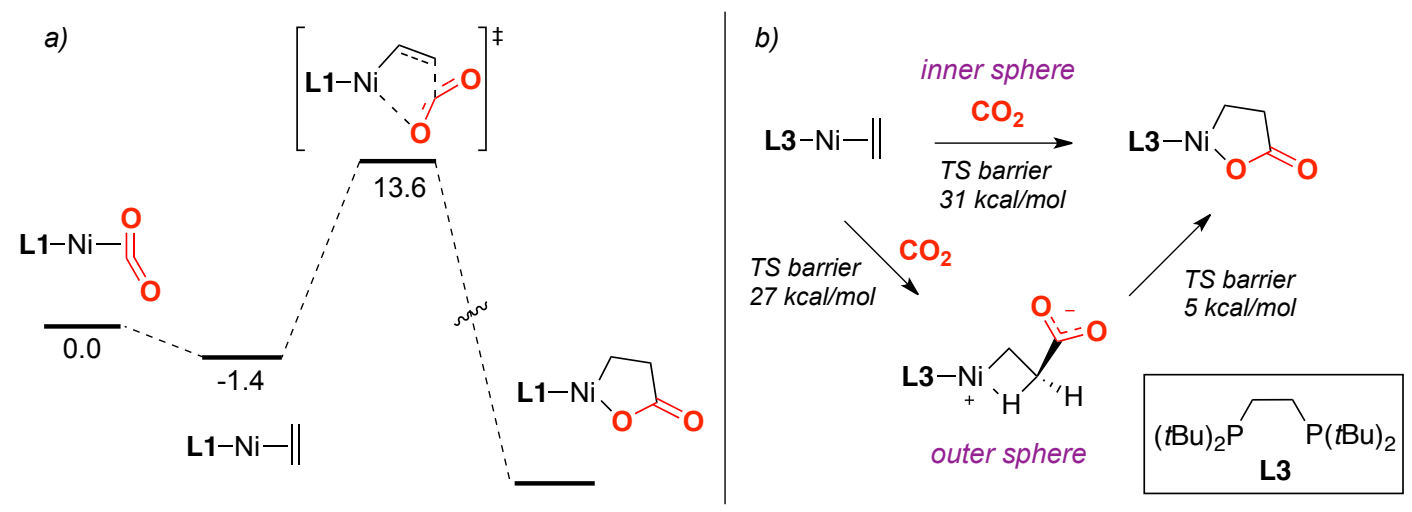

Scheme 15. DFT calculations on the formation of nickelalactones derived from $\mathrm{C}_{2} \mathrm{H}_{4}$ and $\mathrm{CO}_{2}$. Energy levels are expressed in $\mathrm{kcal} / \mathrm{mol}[69,71]$

Although saturated aliphatic carboxylic acids were within reach from the corresponding nickelalactones, stoichiometric functionalizations of these metallacycles should in principle not be limited to protonolysis events. In 1986, Hoberg disclosed that the thermal decomposition of the DBUcontaining nickelacycle derived from the oxidative coupling of styrene and $\mathrm{CO}_{2}$ led to the formation of cinnamic acid (Scheme 16) [62]. The rationale behind this result was interpreted on the basis of a $\beta$-hydride elimination from the corresponding nickelalactone, leading to a $\mathrm{Ni}(\mathrm{II})$-hydride that adds into the $\mathrm{C}=\mathrm{N}$ double bond of the $\mathrm{DBU}$ ligand, affording the corresponding unsaturated carboxylic acid 
after hydrolysis (Scheme 16). This singular discovery opened up new vistas for preparing industrially attractive acrylate derivatives from $\mathrm{CO}_{2}$ and simple olefins (see section 3.2.2).

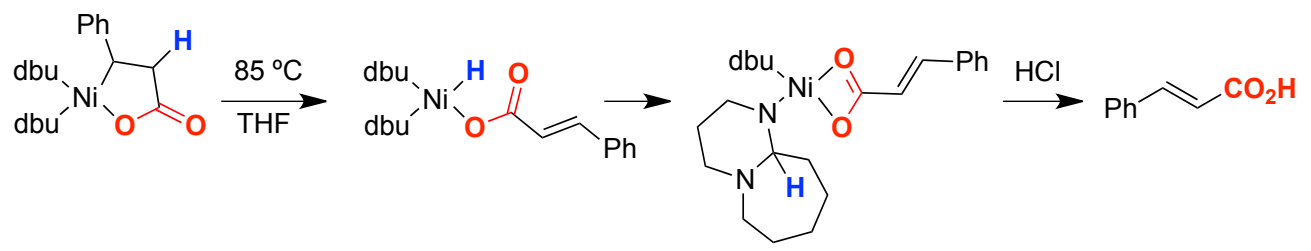

Scheme 16. Cinnamic acid formation via $\beta$-hydride elimination [62]

This rather intriguing reactivity could be turned into a strategic advantage by implementing tandem processes involving the insertion of unsaturated molecules prior the $\beta$-hydride elimination step. In this manner, a variety of carboxylic acids of different nature were prepared in a stoichiometric manner (Scheme 17) [72].

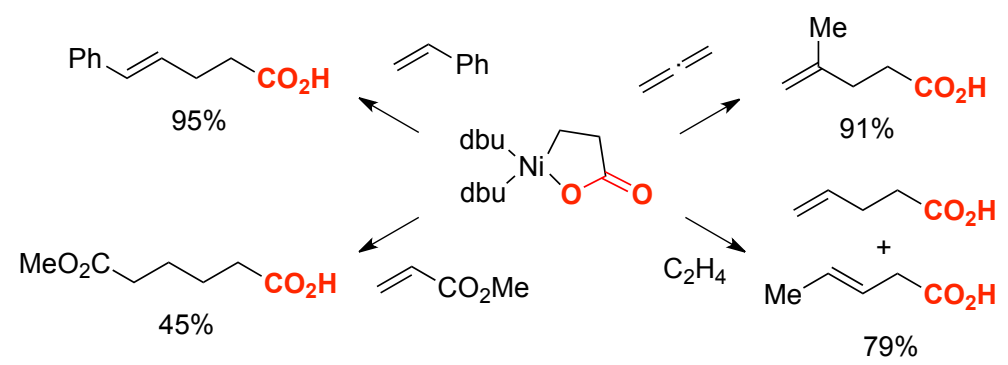

Scheme 17. C-C bond-forming events with nickelalactones [72]

While the cycloaddition of alkenes and $\mathrm{CO}_{2}$ has primarily been conducted with $\mathrm{Ni}(0)$ complexes, it is worth mentioning that a similar reactivity has been observed with $\mathrm{Fe}(0)$ species, forming 5membered metallalactones in the presence of $\mathrm{CO}_{2}$ and ethylene [73]. However, the corresponding oxaferracyclopentanones are rather unstable and their isolation could not be unambigiously confirmed, certainly a serious limitation when compared with their Ni analogues (Scheme 18). In sharp contrast with their nickelalactone congeners, the oxaferracyclopentanones did not result in monocarboxylic acids upon simple protonolysis, but rather in structures bearing two carboxylic acids. Notably, the formation of linear of $\alpha$-branched products was strongly dependent on the bulkiness of the phosphine ligand utilized. For example, while linear bis-carboxylic acids were obtained when operating under a $\mathrm{PMe}_{3}$ regime, a protocol based upon $\mathbf{L 1}$ resulted in a $\beta$-hydride elimination/migratory insertion scenario, leading to the branched product after a final $\mathrm{CO}_{2}$ insertion. 


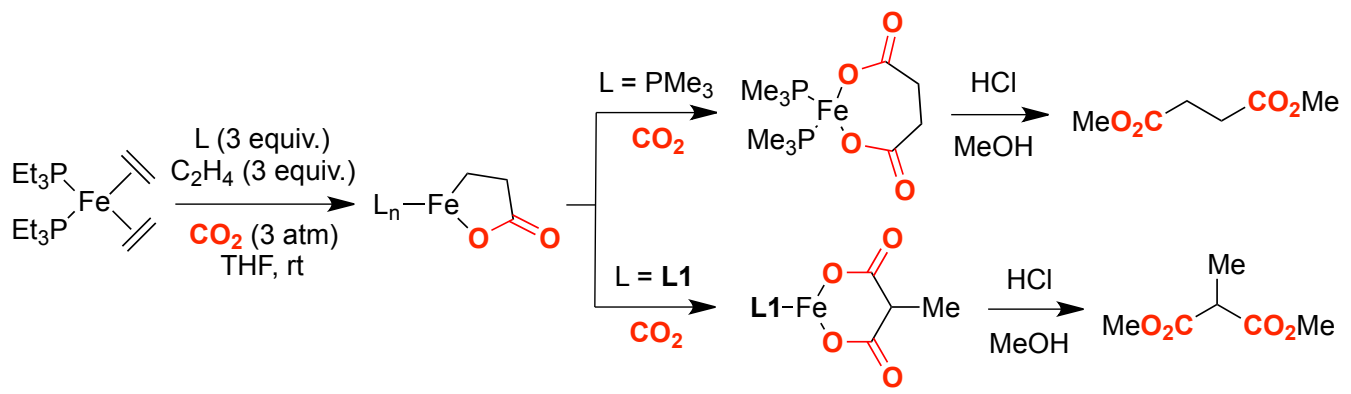

Scheme 18. Ligand-controlled carboxylation of oxaferracyclopentanones [73]

\subsection{Catalytic carboxylation of olefins with $\mathrm{CO}_{2}$}

Despite the elegant stoichiometric studies reported in the 80's and 90's, the catalytic preparation of carboxylic acids via the coupling of alkenes and $\mathrm{CO}_{2}$ still remains challenging. This is likely attributed to the intrinsic inertness of $\mathrm{CO}_{2}$, making the reaction energetically endergonic and kinetically disfavored [74]. Although this issue has been partially overcome with the activation of both counterparts in oxidative cyclization pathways mediated by selected metals ( $\mathrm{Ni}, \mathrm{Fe}, \mathrm{Co}$ ), the corresponding metallacycles are remarkably stable, hence preventing the implementation of a catalytic process. Since the first report of nickelalactones, the vast majority of research has been conducted with the aim of enabling a catalytic carboxylation of olefinic counterparts. At present, two main strategies have been reported: (1) the preparation of saturated carboxylic acids via formal hydrocarboxylation of an olefin with $\mathrm{CO}_{2}$, and (2) the synthesis of acrylate derivatives via $\beta$-hydride elimination of in situ generated nickelelactone intermediates.

\subsubsection{Catalytic reductive carboxylation of alkenes with $\mathrm{CO}_{2}$}

Although this review is focused on carboxylation processes involving $\mathrm{Ni}$ and $\mathrm{Fe}$ complexes, it is worth mentioning that the first report dealing with a catalytic hydrocarboxylation of alkenes was reported in 1978 by Lapidus et al. [75]. The reaction involved homogeneous and heterogeneous Rh and Pd catalysts, which were capable to carboxylate ethylene with $\mathrm{CO}_{2}$ under high pressure and temperature $\left(700 \mathrm{~atm}\right.$ and $\left.180{ }^{\circ} \mathrm{C}\right)$ in the presence of mineral acids. Under these conditions a $38 \%$ yield of propionic acid could be obtained along with ethanol and ethyl propionate as main byproducts. Unfortunately, the authors did not explicitly indicate the catalyst loading utilized, thus leading to a reasonable ambiguity regarding the turnover numbers of this transformation. Apart from this isolated example, a rather limited number of methodologies have been shown to effectively carboxylate olefins with the direct participation of $\mathrm{CO}_{2}$ in a reductive manner. In 2008, Rovis reported an elegant Nicatalyzed direct hydrocarboxylation of styrenes to afford the corresponding $\alpha$-methyl arylacetic acids in a highly regioselective manner [76]. The reaction proceeded at room temperature in the presence of $\mathrm{Ni}(\mathrm{II})$ and $\mathrm{Ni}(0)$ precatalysts and using $\mathrm{ZnEt}_{2}$ as a formal reducing agent (Scheme 19). A variety of electron-neutral and deficient styrenes with different substituent at the aryl moiety including ethers, ester and ketones could be carboxylated in moderate to good yields. Under these conditions, electron- 
rich or $\beta$-substituted styrenes, as well as aliphatic $\alpha$-olefins showed to be almost completely unreactive, thus showing the inherent limitations of this protocol.
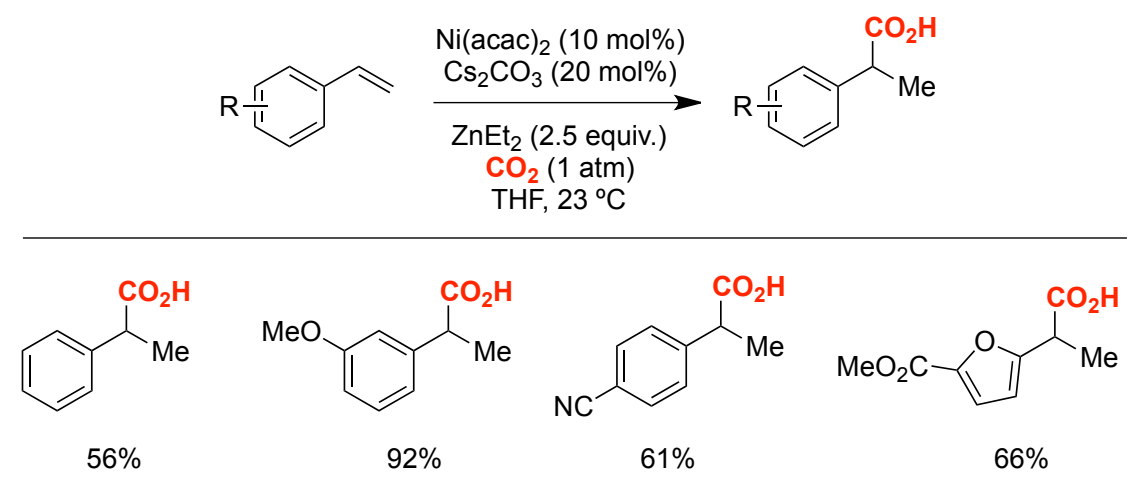

Scheme 19. Ni-catalyzed hydrocarboxylation of styrenes with $\mathrm{Et}_{2} \mathrm{Zn}[76]$

Taking into consideration the experimental results, the authors proposed a mechanistic scenario not consisting of the intermediacy of nickelalactones. Specifically, the authors proposed the participation of $\mathrm{Ni}$ (II) hydride species, which promote a rapid migratory insertion into the styrene backbone giving rise to a benzyl nickel intermediate. Direct carboxylation of the latter with $\mathrm{CO}_{2}$ leads to the formation of the corresponding $\alpha$-branched carboxylate nickel complex (Scheme 20, path a). Catalytic turnover might be effected upon treatment with $\mathrm{Et}_{2} \mathrm{Zn}$ via $\beta$-hydride elimination or via transmetallation of the benzyl nickel species with $\mathrm{Et}_{2} \mathrm{Zn}$. This possibility was supported by deuterium incorporation at the benzylic position on the reduced product when quenching the reaction with $\mathrm{D}_{2} \mathrm{O}$ at short reaction times.
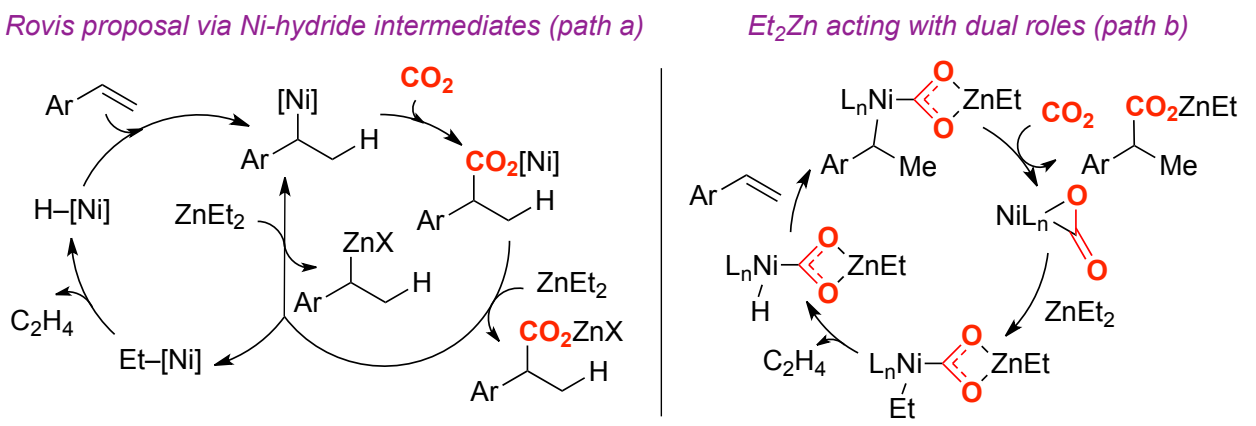

Scheme 20. Mechanism of the Ni-catalyzed hydrocarboxylation of styrenes [76,77]

Recently, the mechanism of this transformation has been studied in detail from a theoretical standpoint. Lin and Yuan compared the energetic profile of the carboxylation reaction via nickelalactone formation and Rovis' suggested nickel hydride intermediates (Scheme 20, path b) [77]. The in-silico study revealed that the generation of a nickelalactone intermediate correlated to a thermodynamic sink, avoiding catalytic turnover. The involvement of nickel hydride species was slightly more favorable energetically, with a significantly lower energetic difference for electron-rich substrates, an observation that could explain their lack of reactivity. As for the nickel hydride 
pathway, theoretical calculations revealed that $\mathrm{ZnEt}_{2}$ act with dual roles, both as a hydride donor by forming ethyl-Ni(II) intermediate that evolves into the catalytically active nickel hydrides upon $\beta$ hydride elimination, as well as a Lewis acid for activating $\mathrm{CO}_{2}$ and therefore facilitating its coordination to the Ni center. In contrast to Rovis' proposal, theoretical calculations point at the formation of the corresponding carboxylic acid via reductive elimination of a $\mathrm{CO}_{2}$-coordinated carboxylate benzyl nickel(II) species. Interestingly, the regioselectivity in the hydrometallation step was studied, revealing that the formation of nickel benzyl species is favored by $4 \mathrm{kcal} / \mathrm{mol}$ when compared to the corresponding phenylethyl derivatives.

Prompted by the seminal work of Rovis on the Ni-carboxylation of styrenes [76] as well as by the work of Hayashi and Shirakawa on the $\mathrm{Fe}$ - and $\mathrm{Cu}$-catalyzed hydromagnesiation of terminal alkenes [78], Thomas reported the preparation of $\alpha$-methyl arylacetic acids in the presence of cheap and bench-stable iron/bis(imino)pyridine catalyst and Grignard reagents at room temperature (Scheme 21). [79] Unlike the Rovis protocol, this reaction turned out to be particularly efficient for styrene derivatives bearing electron-donating groups. However, the reactivity achieved did not seem to be exclusively dictated by electronic mesomeric factors, as 3-methoxystyrene (electron-poor substrate according to its known $-\mathrm{I}$ inductive effect of methoxy groups at the meta position: $\sigma_{\text {meta }}=+0.12$ ) smoothly afforded the corresponding phenyl acetic acid. Unfortunately, the highly reactive nature of Grignard reagents made this methodology incompatible with the use of ketones, esters and other sensitive functional groups. Additionally, non-negligible amounts of the corresponding linear carboxylic acids were observed in certain cases, resulting in a selectivity switch when using cyclopentylmagnesium bromide $\left(\mathrm{Cp}^{\prime} \mathrm{MgBr}\right)$ as reducing agent and ortho-substituted styrenes as substrates. Unfortunately, and in line with Rovis'protocol [76], substitution at the $\alpha$ or $\beta$ positions of the styrene backbone was not tolerated.

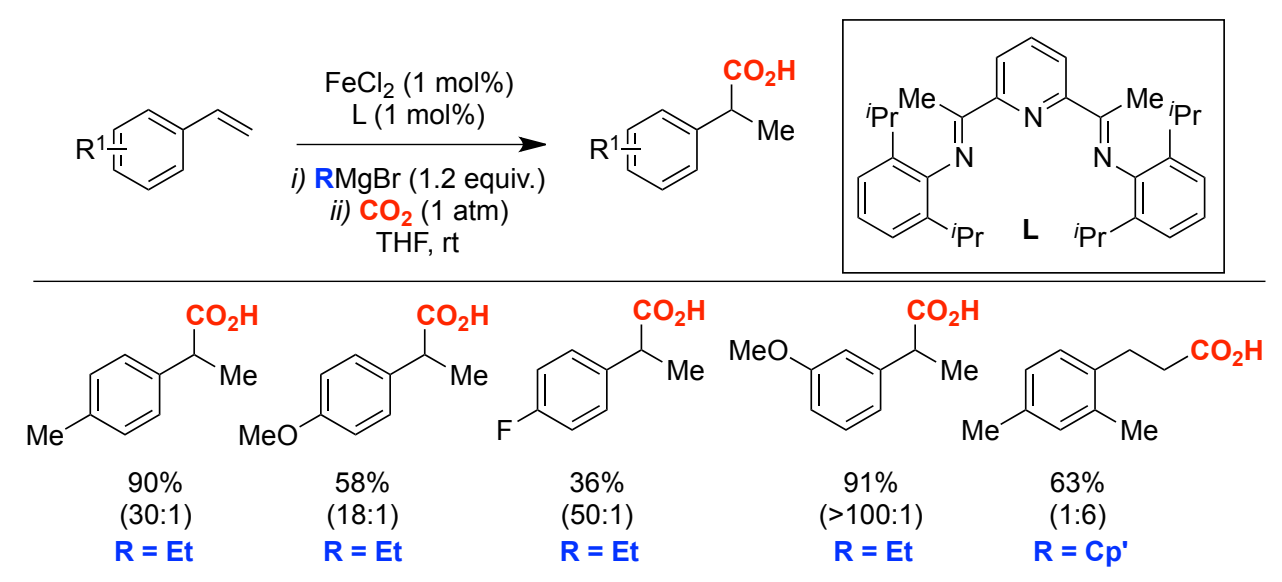

Scheme 21. Fe-catalyzed hydrocarboxylation of styrenes [79]

Quenching experiments with deuterated solvents revealed deuterium incorporation at the benzylic position, thus pointing towards the formation of iron hydride intermediates that trigger an initial 
migratory insertion into the styrene motif followed by transmetallation with the Grignard reagent. The corresponding in situ generated benzylmagnesium bromide derivatives would then react rapidly with $\mathrm{CO}_{2}$ to afford a magnesium carboxylate that upon acidic workup would generate the targeted phenyl acetic acid. In order to unravel the origin of the branched/linear selectivity, the authors tested the reactivity of phenylethylmagnesium bromide under the reaction conditions. In the presence of the iron catalyst, the linear acid was predominantly formed, suggesting that the $\beta$-hydride elimination/hydrometalation sequence is faster than the isomerization of the iron intermediate to produce the $\alpha$-branched product.

Although beyond the scope of this review, it is worth mentioning that a number of elegant hydroxycarbonylation processes have been reported in which $\mathrm{CO}_{2}$ conveniently serves as a surrogate of toxic carbon monoxide (CO) [80]. These processes make use of the so-called water-gas shift reaction (WGSR) using $\mathrm{CO} / \mathrm{H}_{2} \mathrm{O}$ and $\mathrm{CO}_{2} / \mathrm{H}_{2}$ pairs [81]. A few number of reductive carboxylation of olefins following a reverse water-gas shift reaction ( $r$ WGSR) principle has been achieved, especially in the presence of Rh [82]. Recently, the hydroxycarbonylation of styrenes and aliphatic olefins has been addressed by in situ generation of $\mathrm{CO}$ from $\mathrm{CO}_{2}$ and $\mathrm{MeOH}$, using Ni [83] and Ru catalysts [84]. However, these techniques still suffer from the use of harsh conditions, high temperatures and pressures, as well as moderate branched/linear selectivities with aliphatic monosubstituted alkenes.

\subsubsection{Carboxylation of ethylene with $\mathrm{CO}_{2}$ en route to acrylate derivatives}

The direct conversion of alkenes and $\mathrm{CO}_{2}$ into the corresponding $\alpha, \beta$-unsaturated carboxylic acids has been a major research topic in both academic and industrial laboratories [85]. Such interest is primarily associated to the possibility of transforming cheap and abundant chemical feedstocks such as ethylene and $\mathrm{CO}_{2}$ into acrylate derivatives, which rank amongst one of the most versatile building blocks used in chemical industry. After more than 30 years of intense research, however, this transformation is still considered one of the main challenges in modern synthetic chemistry [86].

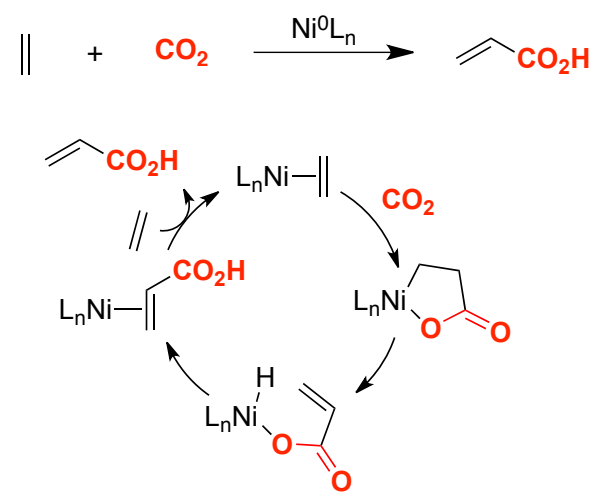

Scheme 22. Hypothetical catalytic cycle for the formation of acrylates

Prompted by the observation that nickelalactones can readily be formed from ethylene and $\mathrm{CO}_{2}$ in the presence of a $\mathrm{Ni}(0) \mathrm{L}_{\mathrm{n}}$ complex, a reasonable pathway for the catalytic production of acrylates 
consists of a subsequent $\beta$-hydride elimination leading to carboxylate nickel hydride species that would deliver the corresponding acrylic acid after reductive elimination and ligand exchange with ethylene (Scheme 22). Although a seemingly trivial transformation, there are a number of daunting challenges associated to this approach: 1) the direct transformation of ethylene and $\mathrm{CO}_{2}$ into acrylic acid is endergonic by more than $20 \mathrm{kcal} / \mathrm{mol}$, therefore making the reaction thermodynamically uphill [74]; 2) the activation barrier for $\beta$-hydride elimination step is energetically very costly (ca. 40 $\mathrm{kcal} / \mathrm{mol}$ ), resulting in a non-favorable kinetic profile [87] and 3) only a few number of ligands with specific features are able to efficiently promote the oxidative cyclization step en route to the key nickelalactone intermediates. Not surprisingly, the vast majority of efforts conducted in this field of expertise have been focused on adjusting the kinetics of the $\beta$-hydride elimination step.

Walther systematically studied the behavior of a number of bidentate phosphine ligands in the formation of acrylate derivatives from five-membered nickelalactone intermediates [88]. Specifically, bisdiphenylphosphine ligands with aliphatic bridges of different lengths were found to be particularly efficient for promoting the targeted reaction (Scheme 23). While the reaction of the TMEDAcontaining nickelalactone with 1,2-bis(diphenylphosphino)ethane (dppe) cleanly gave rise to the corresponding nickelalactone, an intriguing reductive decomposition efficiently occurred with 1,3bis(diphenylphosphino)propane (dppp) and 1,4-bis(diphenylphosphino)butane (dppb), thus forming the corresponding 18-electron $\mathrm{Ni}(0) \mathrm{L}_{2}$ complex. Interestingly, the utilization of a more labile ligand such as bis(diphenylphosphino)methane (dppm) resulted in the formation of a stable $\mathrm{Ni}(\mathrm{I})-\mathrm{Ni}(\mathrm{I})$ dimer containing an acrylate ligand, thus demonstrating the viability for performing a $\beta$-hydride elimination.

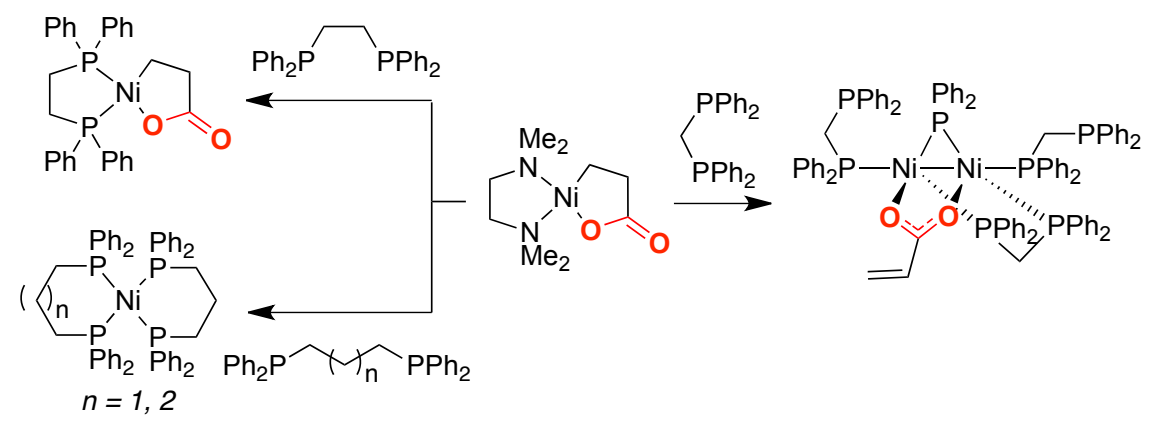

Scheme 23. Bisdiphenylphosphine ligands in the production of acrylates [88]

Encouraged by these results, DFT calculations were performed to study the viability of a catalytic preparation of acrylates from ethylene and $\mathrm{CO}_{2}$, revealing a rate-determining $\beta$-hydride elimination pathway [87]. Interestingly, a distortion-interaction analysis showed a tremendous energetic cost associated with the difficulty of the system to adopt a conformation that could allow a Ni-H agostic interaction, which is crucial for the success of the $\beta$-hydride elimination step. Therefore, a Ni-O bond elongation in the nickelalactone intermediate was predicted to facilitate this step by considerably reducing the ring strain. This crucial information was taken by Rieger, who based their strategy on 
breaking the $\mathrm{Ni}-\mathrm{O}$ bond in the nickelalactone intermediate to promote the $\beta$-hydride elimination process via in situ alkylation of the carboxylate moiety [89]. When treating the corresponding dpppnickelalactone with methyl iodide, a 33\% yield of the methyl acrylate could be detected. IR and NMR monitoring experiments showed the cleavage of the $\mathrm{Ni}-\mathrm{O}$ bond during the reaction and the formation of methyl acrylate. This was further corroborated by the detection of methyl acrylate after the reaction of methyl 3-iodopropionate with the $\mathrm{Ni} / \mathrm{dppp}$ system, which gives rise to the same intermediate. Based on these results, Kühn anticipated that the reaction with methyl iodide should be ligand-dependent [90]; as expected, this turned out to be the case and the ligand utilized exerted a tremendous influence on the formation of methyl acrylate. Among these, TMEDA proved to be superior than dppp and dppe; interestingly, no reaction was observed when exposing nickelalactones bearing dppb or pyridine-containing ligands. Although the corresponding alkyl Ni(II) iodide intermediate formed upon treatment with MeI could be detected by both FT-IR or NMR spectroscopy, its isolation proved to be particularly recalcitrant (Scheme 24). Importantly, DFT calculations revealed that the rate-determining step corresponded to the addition of methyl iodide. In line with this notion, the authors found that the inclusion of methyl triflate (MeOTf) significantly enhanced the rate of the reaction [91]. Unlike the use of methyl iodide, the reaction of MeOTf turned out to be particularly efficient with dppe, dppp or even dppb whereas the employment of TMEDA failed to provide the targeted methyl acrylate. While counterintuitive, this observation could be turned into a strategic advantage, as the corresponding alkyl $\mathrm{Ni}(\mathrm{II})$ triflate intermediate could be isolated and characterized.

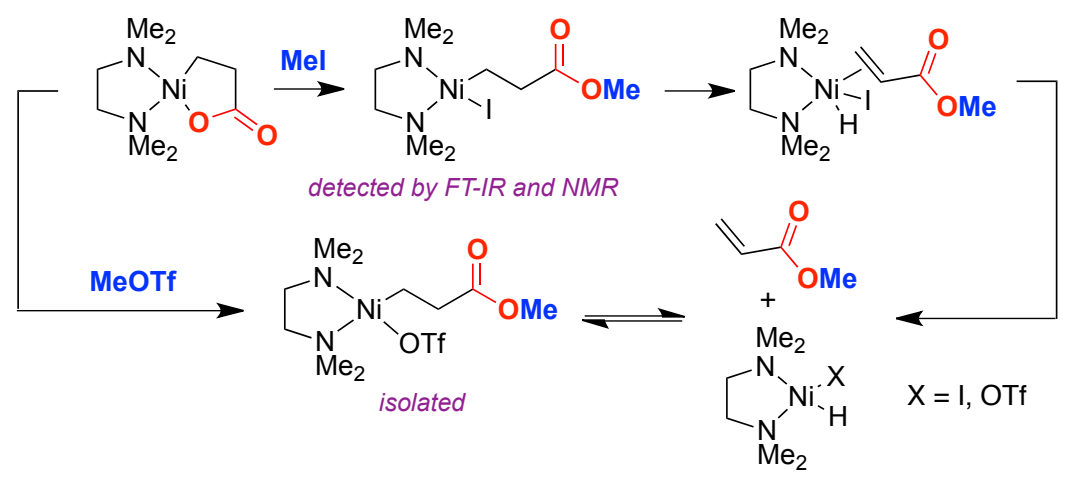

Scheme 24. Nickelalactone ring opening triggered by methyl electrophiles $[90,91]$

Limbach and Hofmann reported a comprehensive theoretical study on the mechanism of the nickelmediated synthesis of methyl acrylate from ethylene, $\mathrm{CO}_{2}$ and methyl iodide [92]. DFT calculations revealed an $\mathrm{SN}_{2}$-type mechanism for the alkylation of the carboxylate ligand, which explain the experimental observations gathered by the groups of Rieger and Kühn. Subsequently, Bernskoetter described the ring-opening of the nickelalactone intermediates by coordination of the carboxylate fragment with Lewis acids [93]. Thus, the reaction of the dppf [bis(diphenylphosphino)ferrocene] nickelalactone with $\mathrm{BAr}_{3}{ }^{\mathrm{f}}$ [tris(pentafluorophenyl)borane] led to the formation of an activated fivemembered nickelacycle ( $\gamma$-nickelalactone), in which the Lewis acid is coordinated to the carboxylate 
fragment, ultimately leading to a four-membered nickelacycle via $\beta$-hydride elimination and subsequent migratory insertion (Scheme 25). The latter species underwent formation of acrylate $\mathrm{Ni}(0)$ $\pi$-complexes upon addition of a base such as BTPP [tert-butyliminotri(pyrrolidino)phosphorane] or DBU. The enhanced selectivity towards the $\beta$-hydride elimination against unproductive pathways in the presence of a base was also studied from a theoretical standpoint [92,94]. Later, the same group demonstrated that the $\gamma$-to- $\beta$ isomerization of nickelalactones could be also achieved with $\mathrm{NaBAr}_{4}{ }^{\mathrm{f}}$, [95] in which the $\mathrm{Na}^{+}$cations were able to lower down the barrier for $\beta$-hydride elimination as well as the energy of other putative reaction intermediates.

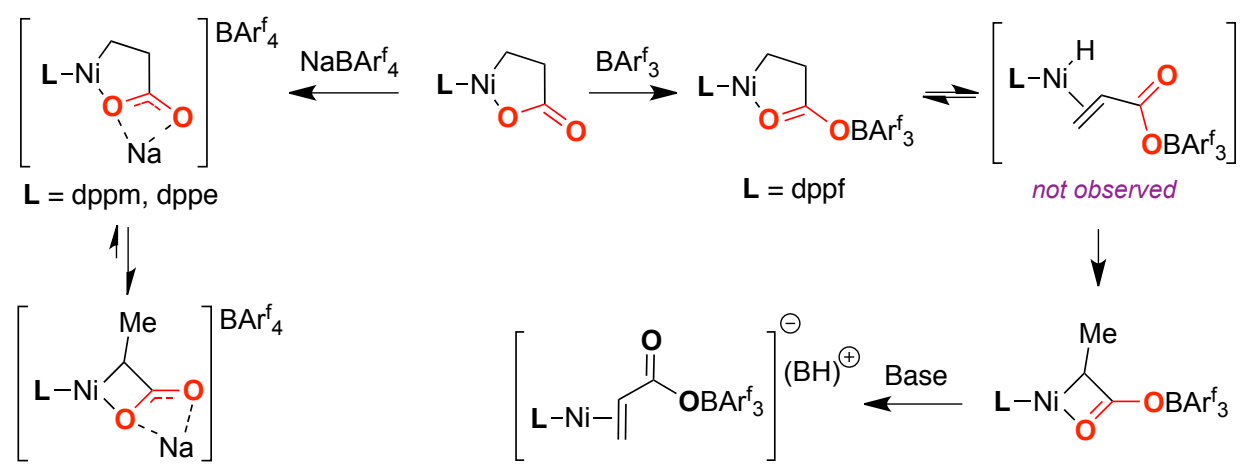

Scheme 25. Ring opening of nickelalactones triggered by Lewis acids $[93,95]$

With all the knowledge acquired from the stoichiometric experiments, Limbach et al. attempted the development of a catalytic carboxylation of ethylene with $\mathrm{CO}_{2}$ using $\mathrm{d} t \mathrm{bpe}$ [1,2bis(ditertbutylphosphino)ethane] as the ligand [96]. The authors showed that exposure of $\mathrm{d} t \mathrm{bpe}$ nickelalactone to either sodium tert-butoxide $(\mathrm{Na} t \mathrm{OBu})$ or sodium hexamethylsidilazide (NaHMDS) resulted in the formation of the acrylate $\mathrm{Ni}(0)$ complex via $\beta$-hydride elimination $(90 \%$ and $87 \%$ yield, respectively). Interestingly, while ligand exchange of the acrylate $\mathrm{Ni}(0)$ complex with ethylene occurred effectively at high pressure of ethylene (8-30 bar), thus releasing sodium acrylate, no ligand exchange was observed with acrylic acid. These experiments demonstrated the feasibility of preparing acrylate derivatives from ethylene and $\mathrm{CO}_{2}$ (Scheme 26).

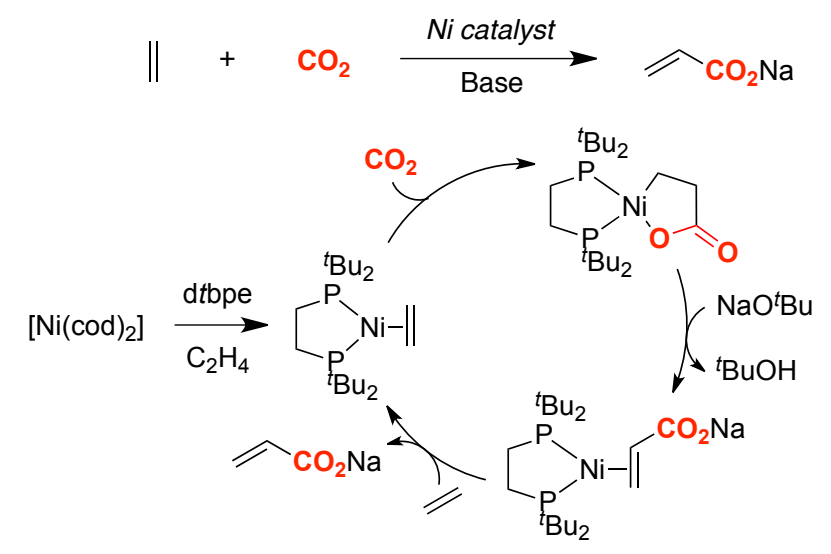

Scheme 26. Catalytic carboxylation of ethylene and $\mathrm{CO}_{2}$ to produce sodium acrylate [96] 
Although Limbach demonstrated the feasibility of all elementary steps within the catalytic cycle, the implementation of a fully integrated catalytic process was far from trivial. Indeed, while nickelalactone formation had to be prepared under a high pressure of $\mathrm{CO}_{2}$, the $\beta$-hydride elimination and ligand exchange needed to be conducted in the absence of $\mathrm{CO}_{2}$ to avoid the formation of carbonic acid half-esters. Still, the authors developed an iterative regime at high and low pressure of $\mathrm{CO}_{2}$ that allowed reaching ten catalytic turnovers, thus constituting the first catalytic reaction of $\mathrm{CO}_{2}$ and ethylene to prepare acrylate derivatives. Recently, Schaub et al. discovered that formation of carbonic acid half-esters could be avoided by increasing the temperature up to $145^{\circ} \mathrm{C}$, successfully obtaining catalytic turnover for the non-iterative carboxylation process [97]. In 2014, Pidko exhaustively studied this catalytic transformation in the presence of different bidentate phosphine ligands using DFT calculations [70]. Although this study confirmed the important role of the ligand on nickelalactone formation, there was a marginal electronic, geometric or steric effect of the ligand on the catalytic activity. In line with the experiments performed by Limbach, it was found that the energy barrier for $\beta$-hydride elimination was particularly low when employing $\mathrm{NaOMe}$, due to a fast deprotonation and an increased stabilization of the putative intermediates by the presence of $\mathrm{Na}^{+}$.

Undoubtedly, the need for an iterative technique at high and low pressure of $\mathrm{CO}_{2}$, together with the requirement for strong nucleophilic bases, represented an important drawback to be overcome when designing a catalytic production of sodium acrylate from ethylene. The authors found a subtle balance of nucleophilicity and basicity when dealing with sodium 2-fluorophenoxide as the base, efficiently promoting the formation of the acrylate $\mathrm{Ni}(0)$ complex via deprotonation of the corresponding nickelalactone in the presence of $\mathrm{CO}_{2}$ [98]. This result enabled the design of a one-pot catalytic carboxylation of ethylene, styrenes and 1,3-dienes with electron-rich bidentate phosphine ligands. Fine-tuning of the reaction conditions (ligand, Ni catalyst and additives) led to the development of a highly active catalytic procedure, able to promote the carboxylation of ethylene to more than 100 turnover numbers (TON) (Scheme 27). Notably, styrene displayed a moderate reactivity (12 TONs) whereas the inclusion of electron-withdrawing or electron-donating groups resulted in lower TONs. Interestingly, $(E)$-configured acrylic acids were obtained in all cases analyzed.

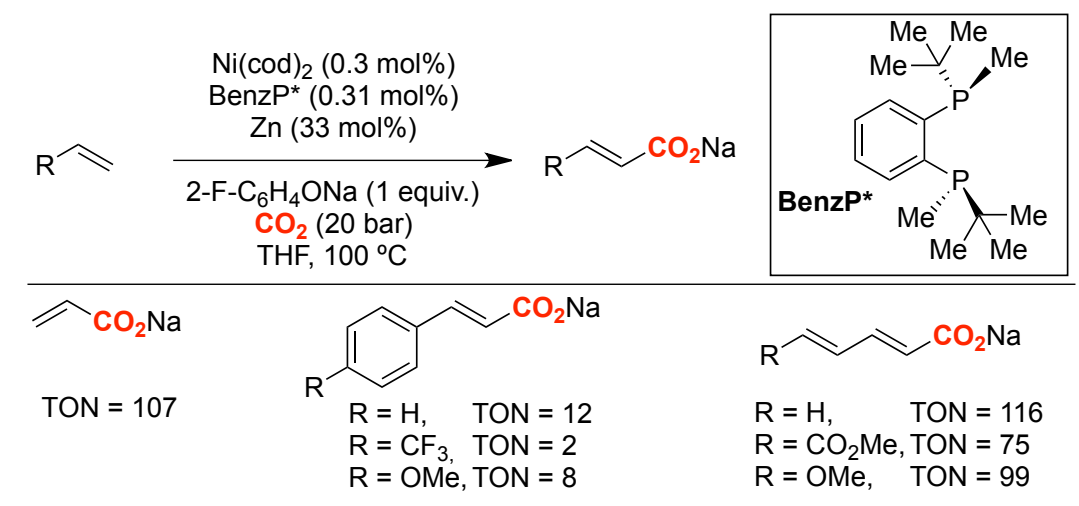

Scheme 27. Catalytic synthesis of acrylates in the presence of sodium 2-fluorophenoxide [98] 
Recently, Schaub has experimentally investigated the mechanism of this transformation and expanded the scope of this reaction to 1,3-dienes [99]. Simultaneously, Vogt reported a different strategy to achieve the catalytic formation of acrylate derivatives from $\mathrm{CO}_{2}$ and ethylene. The authors tackled the critical ring opening of nickelalactone derivatives with the utilization of a hard Lewis acid that could compete with the Ni center for binding the carboxylate moiety. Specifically, the authors could enable the $\beta$-hydride elimination event by adding lithium iodide and triethylamine as base [100]. Furthermore, DFT calculations predicted an improved behaviour of $\mathrm{Li}^{+}$compared to $\mathrm{Na}^{+}$when promoting the ring opening of the nickelalactone followed by $\beta$-hydride elimination. A series of experiments led to the optimal conditions for the carboxylation of ethylene using a regime based on $\mathrm{Ni}(\operatorname{cod})_{2}$, dcpp [1,3-bis(dicyclohexylphosphino)propane] as ligand, LiI, triethylamine and $\mathrm{Zn}$, forming lithium acrylate in chlorobenzene at $50{ }^{\circ} \mathrm{C}$ with a TON of 21. The presence of overstoichiometric amounts of $\mathrm{Zn}$ was indispensable to avoid catalyst deactivation by converting $\left[\mathrm{Ni}(\mathrm{dcpp}) \mathrm{I}_{2}\right]$ to the corresponding propagating active $\mathrm{Ni}(0)$ species. More recently, Schaub has further improved the catalytic preparation of acrylates avoiding the use of stoichiometric $\mathrm{Zn}$ [101], thus univocally demonstrating that this technology is still at its infancy for producing industrially-relevant acrylic acid from ethylene and $\mathrm{CO}_{2}[102]$.

\section{Carboxylation of multiple unsaturated systems}

\subsection{Allenes}

\subsubsection{Stoichiometric reactions using allenes}

In 1984, Hoberg reported the first $\mathrm{Ni}(0)$-mediated carboxylation of allenes with $\mathrm{CO}_{2}$ (Scheme 28) [103]. Interestingly, the reaction turned out to be highly regio- and stereoselective, as illustrated by the fact that a wide variety of compounds were obtained as single products. Specifically, it was found that the reaction proceeds via the formation of the less hindered nickelalactone and that the corresponding $E$-isomer was formed preferentially. Subsequently, Mori reported that DBU could efficiently be used for an otherwise identical transformation [104]. Interestingly, the utilization of 1-silyl-3-alkylsubstituted allenes turned out to be highly advantageous, obtaining high yields of the targeted products and with an excellent regioselectivity profile [105]. In 2004, the Iwasawa group disclosed the use of bidentate bis-amidine ligands in the $\mathrm{Ni}$-mediated carboxylation of allenes, obtaining regioisomeric mixtures of the targeted carboxylic acids, an issue that can be tentatively attributed to the in situ formation of two regioisomeric nickelalactones [37]. 


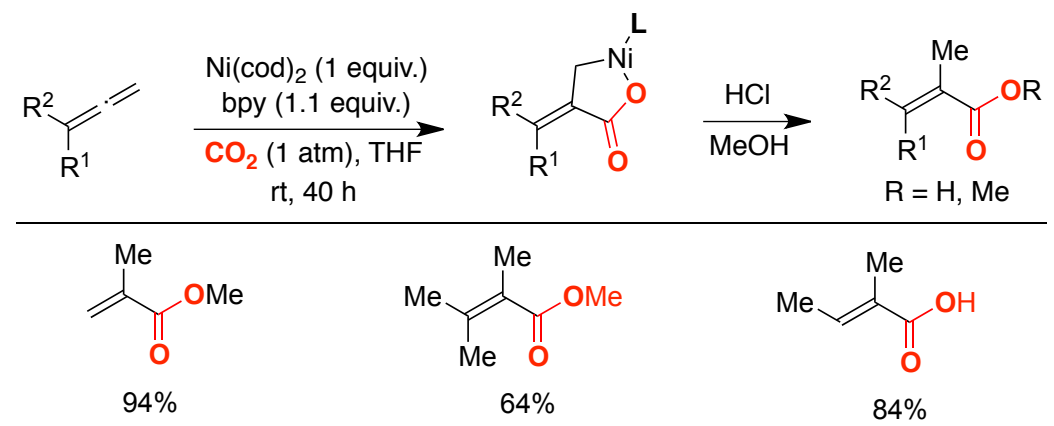

Scheme 28. Ni(0)-mediated carboxylation of allenes reported by Hoberg. [103]

The Mori group demonstrated the viability of trapping the intermediate nickelalactone deriving from an allene, $\mathrm{Ni}(0)$ and $\mathrm{CO}_{2}$ with electrophiles other than a proton source. Specifically, it was found that lactones possessing an exocyclic double bond are within reach upon treatment with aldehydes and a final acidic workup with pyridinium $p$-toluenesulfonate (PPTS) (Scheme 29) [104]. Interestingly, the regioselectivity observed differs from that shown in the absence of $\mathrm{ArCHO}$ upon simple acidic workup, suggesting that the selectivity switch depends predominantly on the electrophile utilized. With all the experimental results in hand, the authors proposed that the observed regioselectivity could be dictated by two alternate pathways dealing with the intermediacy of $\pi$-allyl $\mathrm{Ni}$ intermediate or the formation of a single nickelalactone intermediate that would evolve to the targeted product via ipso substitution. Recently, the Sato group demonstrated the possibility of conducting an otherwise related transformation, but in an intramolecular fashion [106].

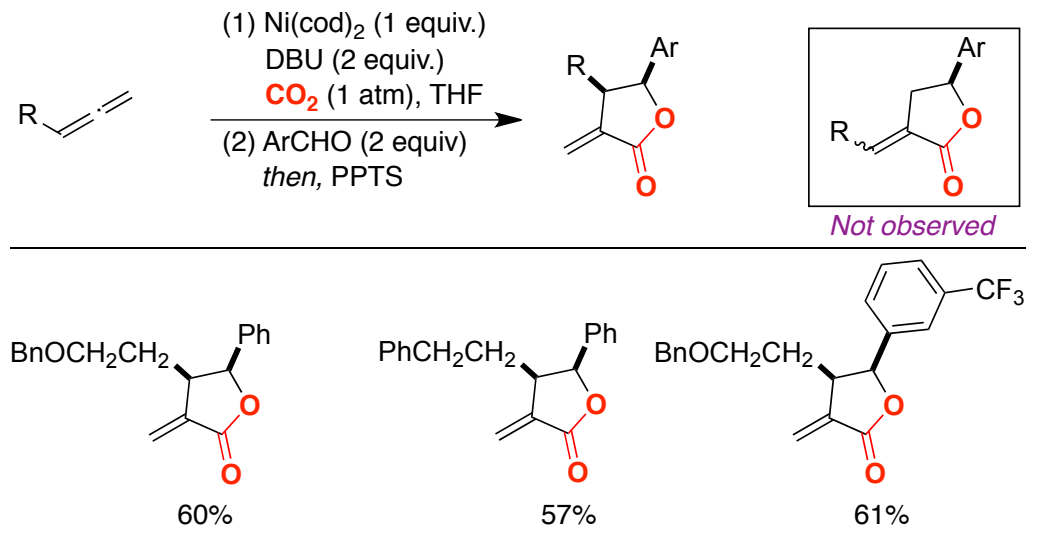

Scheme 29. $\mathrm{Ni}(0)$-mediated lactone formation from allenes and $\mathrm{CO}_{2}[104]$

In 2007, Iwasawa reported an interesting oxidative cleavage of the nickelalactone intermediates utilizing oxygen as oxidizing agent (Scheme 30) [107]. Unlike previous carboxylation of allene intermediates, the reaction resulted in regioisomeric mixtures of allylic alcohols, with a slight preference for the corresponding secondary alcohol. The authors rationalized these results via the involvement of a $\pi$-allyl $\mathrm{Ni}(\mathrm{II})$-intermediate, although it was not possible to rule out an alternative based on the formation of regioisomeric $\sigma$-complexes. 


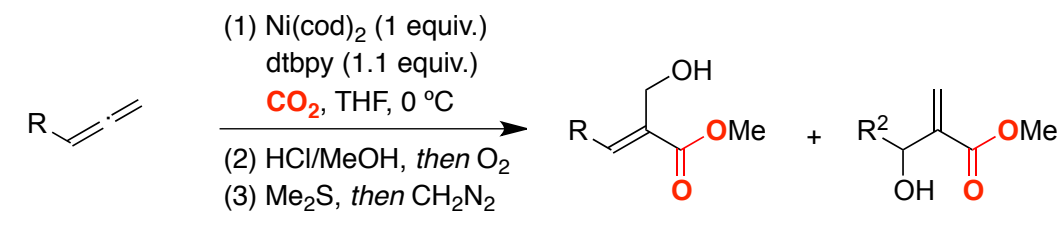

-hex

Scheme 30. Oxidative cleavage of allene-derived nickelalactones using $\mathrm{O}_{2}$ [107]

\subsubsection{Catalytic processes involving allenes as substrates}

Unlike the wealth of literature data on stoichiometric reactions of allenes with $\mathrm{CO}_{2}$, a limited number of Ni-catalyzed protocols have been reported. Prompted by the seminal electrochemical method reported by Duñach [108], Mori and Sato described a Ni-catalyzed carboxylation of 1-silyl-3-alkylsubstituted allenes using $\mathrm{Me}_{2} \mathrm{Zn}$ (Scheme 31) [109]. Interestingly, the transformation occurred with a neat incorporation of two molecules of $\mathrm{CO}_{2}$ into the allene backbone, producing a single regioisomer and with high yields. The observed regioselectivity was in agreement with the initial stoichiometric studies reported by Mori when dealing with silanes [105] and carbonyl-type electrophiles [104], via the intermediacy of $\pi$-allyl intermediates. It was believed that the inclusion of $\mathrm{Me}_{2} \mathrm{Zn}$ triggered a transmetalation event of in situ generated nickelalactone, thus forming a nucleophilic allyl Ni(II)methyl species that trigger a subsequent carboxylation event instead of a reductive elimination event.

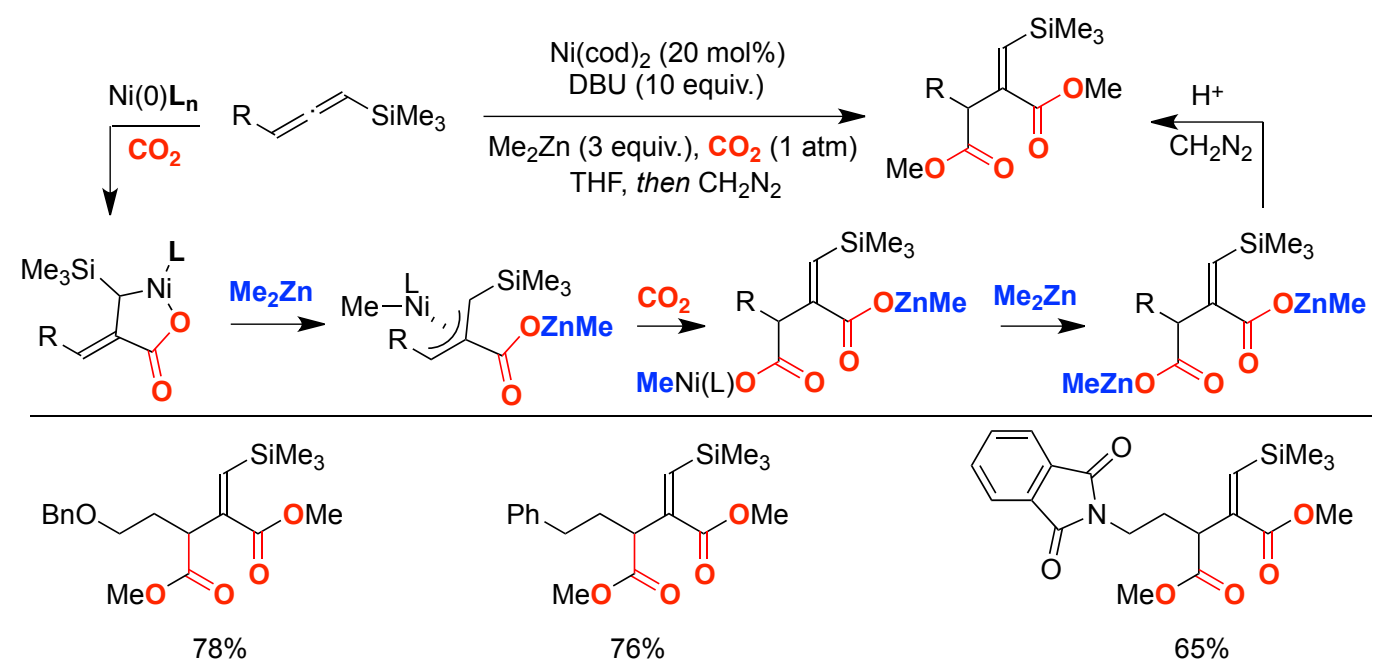

Scheme 31. Ni-catalyzed double carboxylation of allenes [109] 


\subsection{Dienes and related unsaturated systems}

\subsubsection{Stoichiometric studies}

In 1982, Walther disclosed a stoichiometric carboxylation of 2,3-dimethyl butadiene using $\mathrm{Ni}(0)$ with TMEDA as ligand (Scheme 32) [110,111]. The authors isolated and fully characterized by X-ray crystallography a $\pi$-allyl complex that formally consisted of a 1:1:1 adduct of a butadiene derivative, Ni-TMEDA and $\mathrm{CO}_{2}$. Stoichiometric studies revealed that these species could be converted into the targeted carboxylic acids by either treatment with aqueous $\mathrm{H}_{2} \mathrm{SO}_{4}$ or MeI. In the latter case, a formal homologation of the 1,3-diene backbone was achieved, an observation that is consistent with a nucleophilic attack of the more basic $\mathrm{C}-\mathrm{Ni}$ bond to MeI.

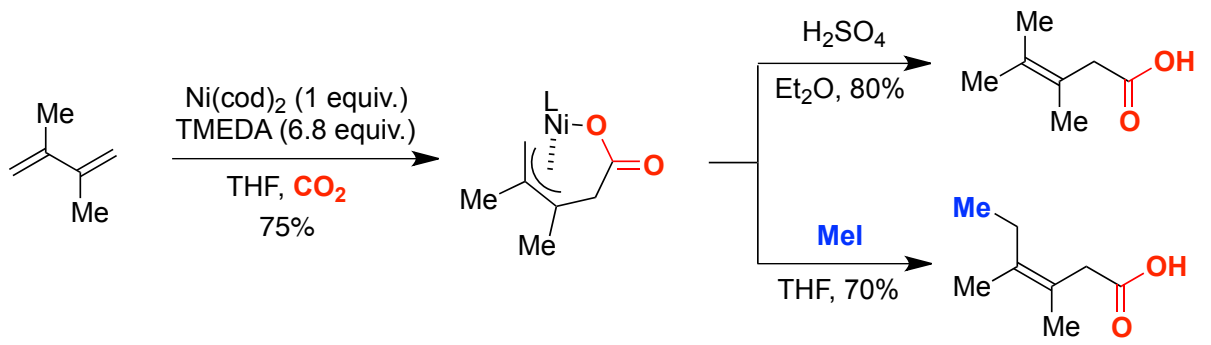

Scheme 32. Walther's studies on the Ni-mediated carboxylation of 2,3-dimethyl butadiene [110,111]

In parallel to these investigations, Hoberg studied a very similar process using butadiene as model substrate (Scheme 33) $[112,113]$. In this case, however, the corresponding $\pi$-allyl $\mathrm{Ni}(\mathrm{II})$ intermediate could not be isolated in its pure form. Indirect evidence for such intermediate, however, was revealed upon quenching with aqueous $\mathrm{HCl}$ and subsequent $\mathrm{MeOH}$ treatment, as the corresponding methyl ester possessing a terminal double bond on the side-chain was exclusively observed. Unlike the Walther method, this protocol ended up in the corresponding terminal alkenes, an observation that is tentatively ascribed to the substitution pattern present on the diene backbone. More interestingly, $(Z)$ dimethyl-3-hexenedioate was found to be within reach via a subsequent $\mathrm{CO}_{2}$ from the nickelalactone intermediate $[113,114]$.

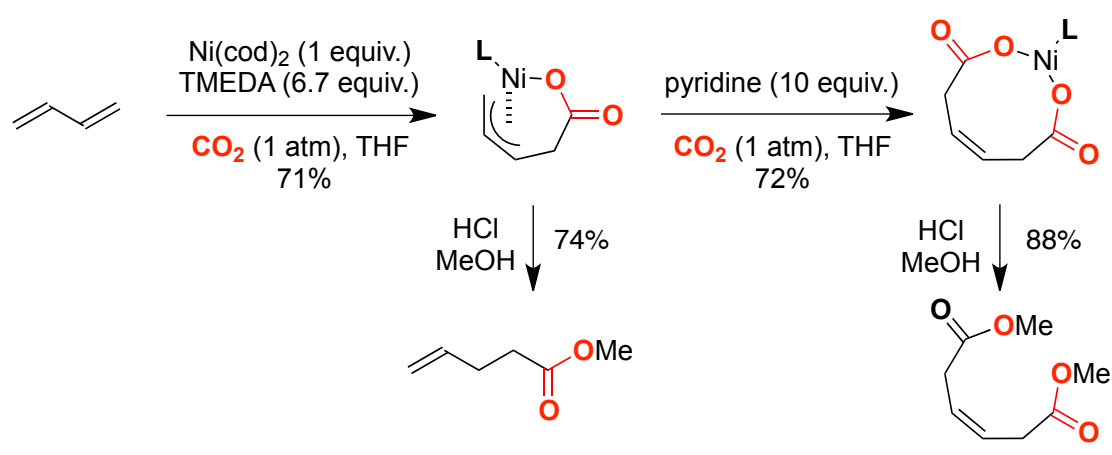

Scheme 33. Hoberg's studies on the Ni-mediated mono- and bis-carboxylation of butadiene [112,113]

Behr and Kanne studied the behavior of trienes bearing both an isolated olefin and a conjugated 1,3diene moiety under similar conditions, demonstrating that the carboxylation event is selective for 1,3- 
dienes and that the isolated olefin does not affect the outcome of the reaction [114]. Hoberg showed that an otherwise identical mono- and bis-carboxylation could also be achieved using Fe-complexes [116]. Kinetic studies by Geyer and Schindler using isoprene as model compound highlighted the importance of the ligands employed, suggesting that future catalytic variants should be focused on ligand design [117]. Furthermore, Saito, Yamamoto and co-workers showed that mono-carboxylation of 1,3-unsaturated backbones can be accomplished using enynes and diynes, in which $\mathrm{CO}_{2}$ insertion occurred at the alkyne terminus and at the less-hindered site [36].

In 2001, a significant step-forward the development of a catalytic methodology was reported by Takimoto and Mori (Scheme 34) [118]. In this work, the authors described that DBU significantly increased the efficiency of the initial $\pi$-allyl $\mathrm{Ni}(\mathrm{II})$ complex. Simple protonolysis of such adduct resulted in a mixture of regioisomers, with slight preference for the carboxylation at the benzylic position. Interestingly, while a cis-configured double carboxylation product was obtained upon treatment with $\mathrm{CO}_{2}$ in the presence of $\mathrm{Me}_{2} \mathrm{Zn}$, the inclusion of $\mathrm{Ph}_{2} \mathrm{Zn}$ delivered a mixture of transconfigured monocarboxylation products. The rationale behind these results was attributed to a change in mechanism; while the second carboxylation event occurs by a $\mathrm{CO}_{2}$ insertion into the allyl $\mathrm{C}\left(\mathrm{sp}^{3}\right)-\mathrm{Ni}$ bond, the presence of $\mathrm{Ph}_{2} \mathrm{Zn}$ triggers a fast transmetallation followed by reductive elimination. Such hypothesis could be corroborated by conducting a reaction with cyclohexadiene as substrate, observing that the double carboxylation with $\mathrm{Me}_{2} \mathrm{Zn}$ gave rise to the trans-product whereas a cisisomer was obtained upon treatment with $\mathrm{Ph}_{2} \mathrm{Zn}$.
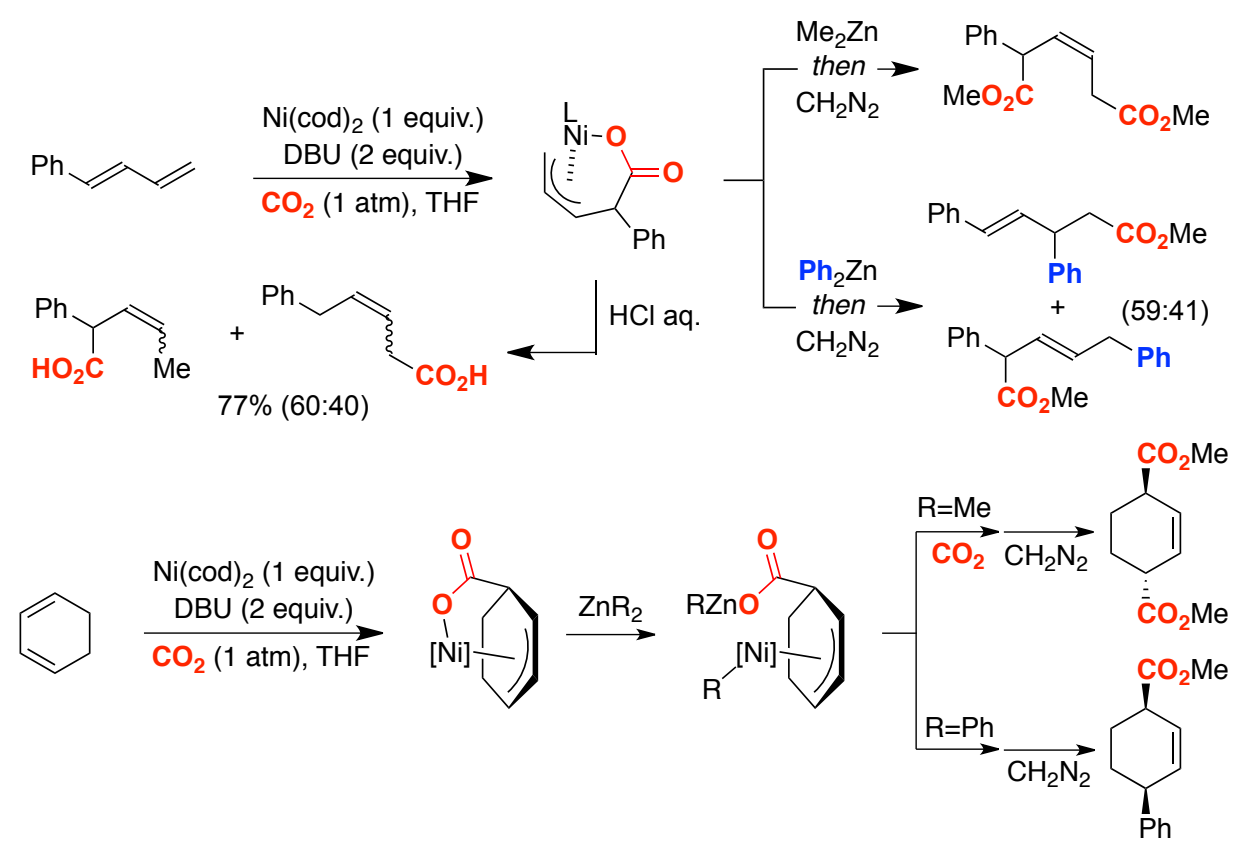

Scheme 34. Reactivity of 1,3-diene: $\mathrm{CO}_{2}$ : $\mathrm{Ni}$ adducts [118].

In 2005, Sato and Mori described the viability of performing a carboxylation of 1,6- and 1,7-enynes mediated by $\mathrm{Ni}(\operatorname{cod})_{2}$ and DBU (Scheme 35) $[119,120]$. Unlike the previous method reported by Mori 
[118], conjugation of the two $\pi$-components was not required; unfortunately, however, the method was limited to a rather specific class of substrates with a Thorpe-Ingold effect for building up five- and six-membered rings, in all cases requiring the presence of electron-withdrawing substituents at the olefin backbone. Still, excellent diastereoselectivities were observed in all cases analyzed, thus setting the stage for the application of this protocol for natural product synthesis as well as for the design of a catalytic process [121].

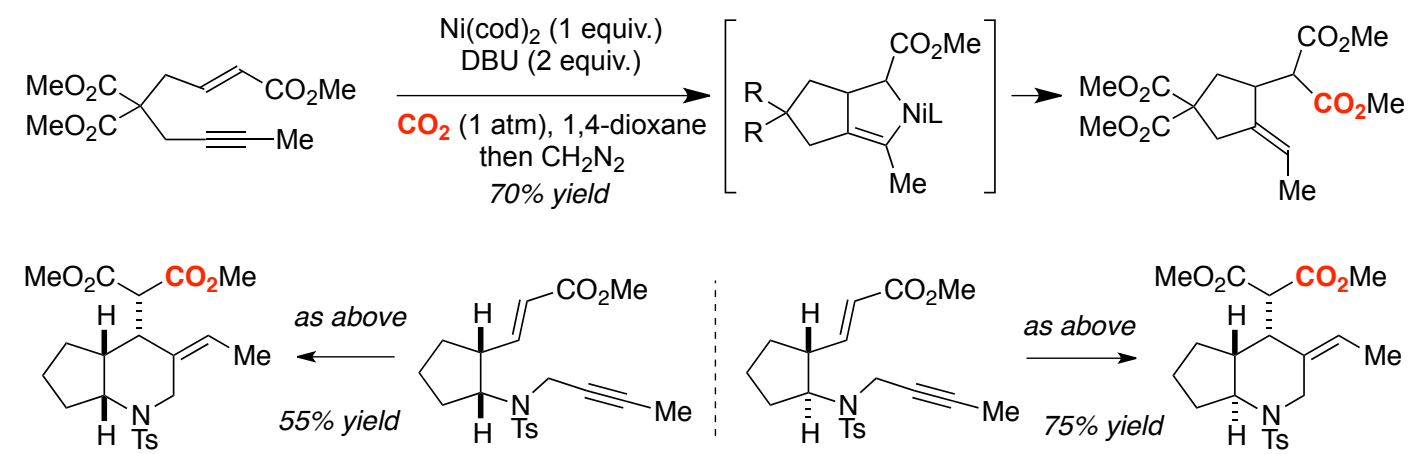

Scheme 35. Ni-mediated cyclization/carboxylation reaction of 1,6-enynes $[119,120]$

\subsubsection{Catalytic carboxylation of 1,3-dienes}

In 1987, Hoberg pioneered the development of a catalytic carboxylation of butadiene using $\mathrm{CO}_{2}$ at high pressures, resulting in cyclopentane carboxylic acids deriving from a dimerization/carboxylation event (Scheme 36) [122]. Although low turnover numbers were obtained, the molecular complexity achieved in this carboxylation event is certainly noteworthy. The postulated catalytic cycle consisted of an initial dimerization of butadiene followed by $\mathrm{CO}_{2}$ insertion into the $\mathrm{C}-\mathrm{Ni}$ bond. The resulting $\pi$ allyl $\mathrm{Ni}(\mathrm{II})$ species triggered an intramolecular carbometalation en route to a six-membered nickelacycle that likely evolves via $\beta$-hydride elimination and a final reductive elimination while recovering back the propagating $\mathrm{Ni}(0)$ species. Unfortunately, no details were indicated regarding the stereochemistry of the final carboxylated product.

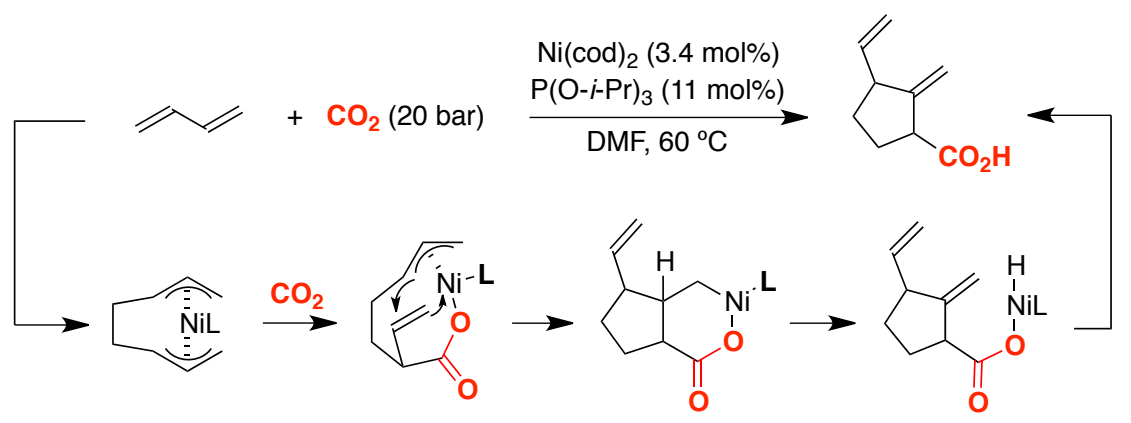

Scheme 36. Ni-catalyzed butadiene dimerization/carboxylation at high pressure $\mathrm{CO}_{2}[122]$

Shortly after, the group of Tsuda and Saegusa reported their studies on the Ni-catalyzed carboxylative cyclization of 1,6- and 1-7-diynes using phosphine ligands at high pressure $\mathrm{CO}_{2}$ (50 bar) $[123,124]$. Unlike the dimerization protocol described by Hoberg, this reaction was proposed to 
proceed through an initial nickelalactone formation with one of the alkyne terminus followed by carbometallation with the pending alkyne, leading to a seven-membered intermediate that ultimately gives rise to a bicyclic $\alpha$-pyrone while regenerating the active $\mathrm{Ni}(0)$ catalyst. While the mechanistic rationale invoked that $\mathrm{CO}_{2}$ insertion occured at the less hindered alkyne, Louie revealed in a subsequent study that the regiochemistry was misassigned and that $\mathrm{CO}_{2}$ insertion occurs adjacent to the most sterically hindered site, consistent with the preferential formation of a nickelalactone with the most sterically hindered alkyne (Scheme 37) $[125,126]$. In contrast to the protocol described by Tsuda and Saegusa, Louie employed $N$-heterocyclic carbene ligands (NHC), enabling to operate at atmospheric pressure of $\mathrm{CO}_{2}$ under exceptional mild conditions. In all cases analyzed, good yields and excellent regioselectivities of the corresponding bicyclic $\alpha$-pyrones were obtained for unsymmetrical diynes, regardless of whether substituents at the tether were present or not. This observation was further corroborated by X-ray crystallographic analysis.

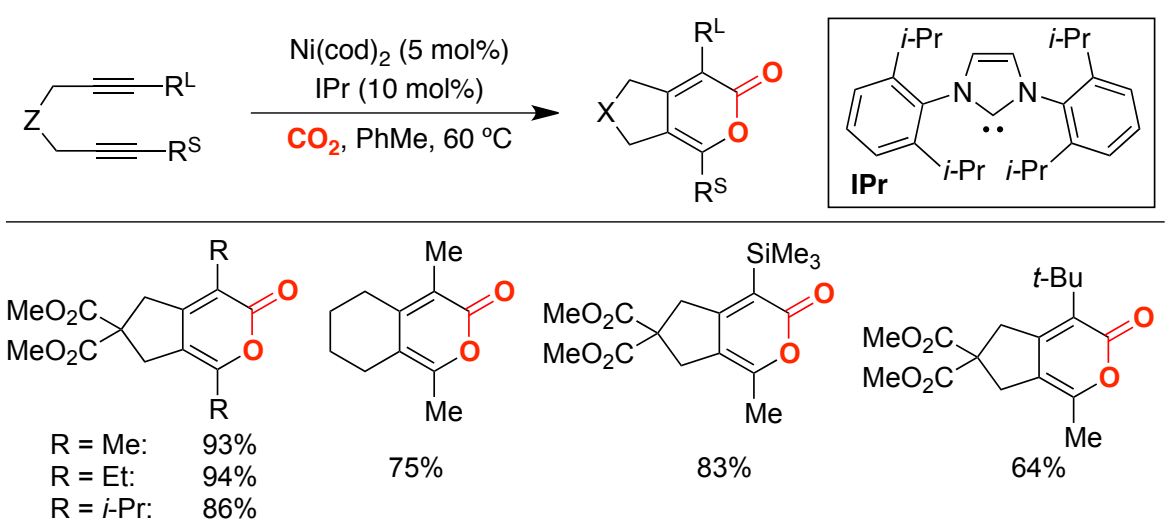

Scheme 37. Ni-catalyzed carboxylative cyclization of 1,6 and 1,7-diynes [125,126]

Very recently, the group of Ma has reported the hydrocarboxylation of similar 1,6- or 1,7-diynes compounds giving rise to the corresponding methyl 2,4-alkadienoate esters after treatment with $\mathrm{CH}_{2} \mathrm{~N}_{2}$ (Scheme 38) [127]. The reaction is remarkably regioselective in the case of diynes containing aliphatic substituents, providing the corresponding $(Z, E)$-carboxylic acids in high yields. However, mixtures of the two possible $(Z, E)$ and $(E, E)$ isomers were found when using aryl substituents, especially bearing electron-withdrawing groups, with preferential formation of the $(Z, E)$-product. After gathering some mechanistic insights, the authors explained the formation of the products via an alkyne-directed hydrocarboxylation pathway. First, the $\mathrm{Ni}(0)$ active species oxidatively inserts into the more electronrich alkyne followed by a transmetalation with $\mathrm{ZnEt}_{2}$. After $\beta$-hydride elimination, the in situ generated $\mathrm{Ni}-\mathrm{H}$ intermediate inserts into the second alkyne leading to Int (Scheme 38.) Finally, $\mathrm{C}-\mathrm{H}$ bond-forming reductive elimination followed by carboxylation of the organozinc species ultimately affords the corresponding products. 


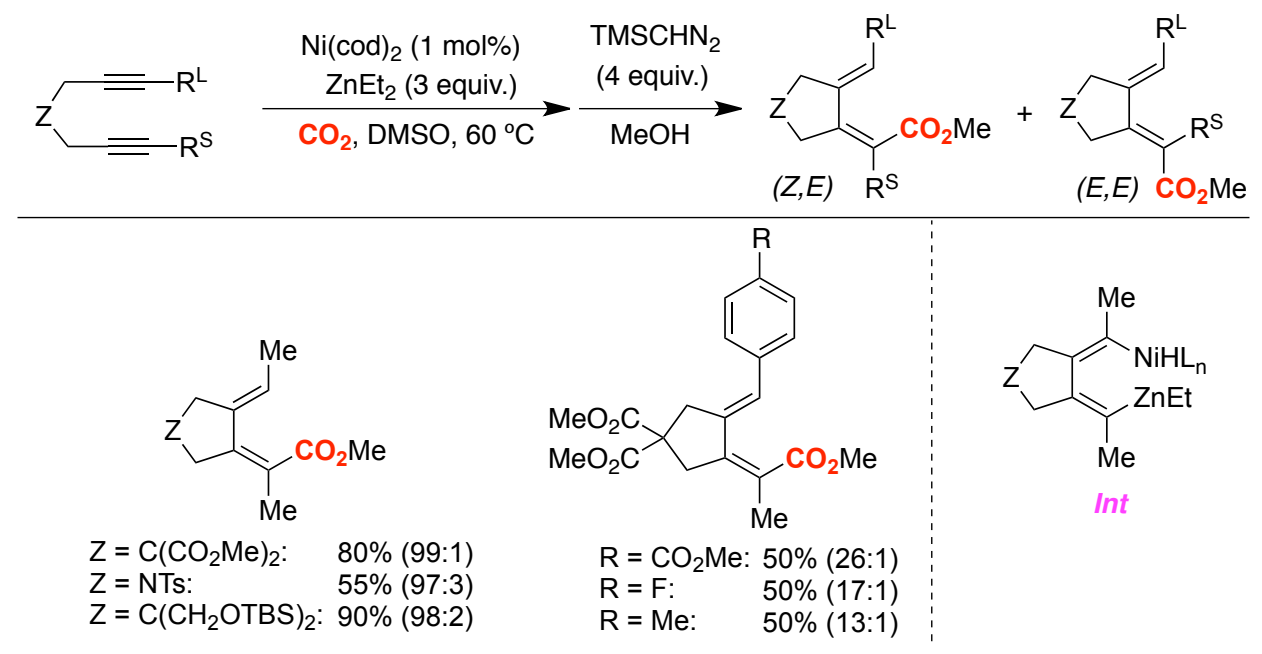

Scheme 38. Ni-catalyzed regioselective hydrocarboxylation of 1,6 and 1,7-diynes [127]

Following up on their previous stoichiometric work, Takimoto and Mori disclosed a Ni-catalyzed carboxylative cyclization of bis(1,3-dienes) possessing substituents at the tether with $\mathrm{CO}_{2}$ and organozinc reagents (Scheme 39) [128]. The reaction was proposed to proceed via initial intramolecular dimerization of two diene moieties, leading to a bis- $\pi$-allyl $\mathrm{Ni}(\mathrm{II})$ intermediate that rapidly reacts with $\mathrm{CO}_{2}$, resulting in a $\pi$-allyl carboxylate $\mathrm{Ni}$ (II) that was inherently disposed to a transmetalation with organozinc species. A final reductive elimination delivers the zinc carboxylate that ultimately is treated with $\mathrm{CH}_{2} \mathrm{~N}_{2}$ to afford the final ester motifs. The high diastereoselectivity observed is inevitably linked to the initial cyclization step that is facilitated by the presence of substituents at the tether. Furthermore, the utilization of unsymmetric bis(1,3-dienes) resulted in a $\mathrm{CO}_{2}$ insertion at the less hindered site. Interestingly, the nature of the organozinc derivative exerted a profound influence on the reaction outcome; while $\mathrm{Me}_{2} \mathrm{Zn}$ and $\mathrm{Ar}_{2} \mathrm{Zn}$ invariably provided good results, competitive $\beta$-hydride elimination was found when employing $\mathrm{Et}_{2} \mathrm{Zn}$.

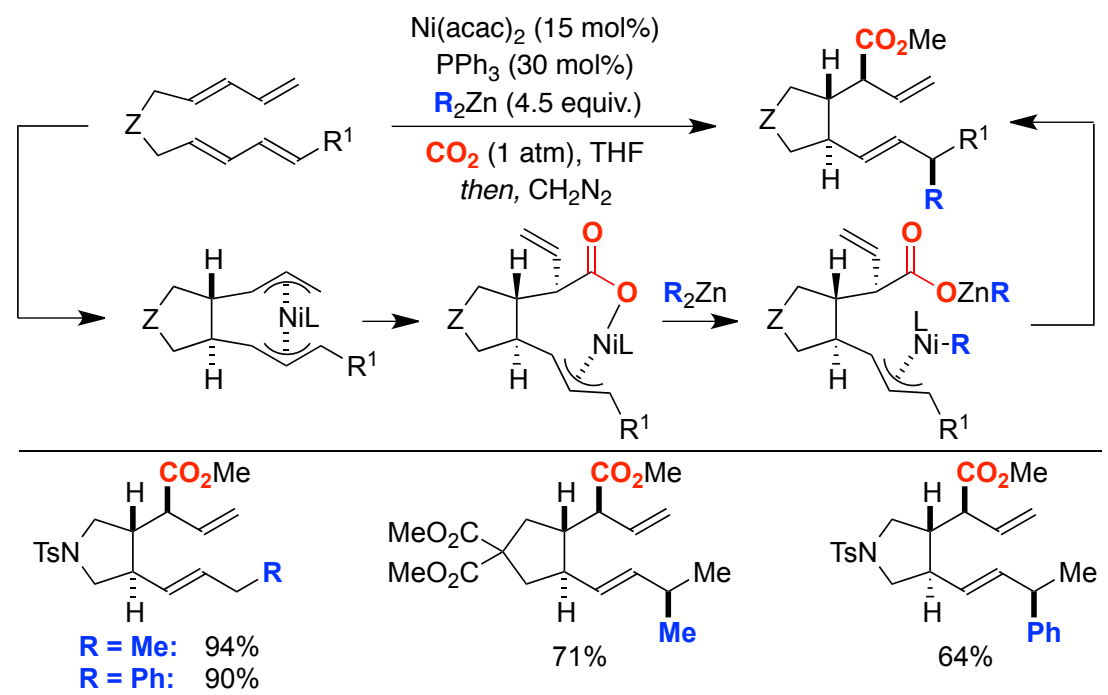

Scheme 39. Ni-catalyzed cyclization/carboxylation of bis(1,3-dienes) [128] 
In 2004, Mori and co-workers expanded the synthetic utility of the carboxylation of bis(1,3-dienes) by designing an enantioselective variant based on a MeO-MOP regime (Scheme 40) [129]. In all cases analyzed, excellent yields and enantioselectivities were obtained for a wide range of substrates. Unlike the corresponding racemic version based on $\mathrm{PPh}_{3}$ (Scheme 39), the authors found that the chiral ligand utilized largely suppressed the parasitic $\beta$-hydride elimination when utilizing $\mathrm{Et}_{2} \mathrm{Zn}$. Interestingly, the reduced product was formed with an otherwise identical degree of enantioinduction, an observation that is in good agreement with the mechanistic rationale highlighted in Scheme 39, thus implying that the pathways leading to both product and reduced species only diverge after the enantiodetermining step has already occurred.

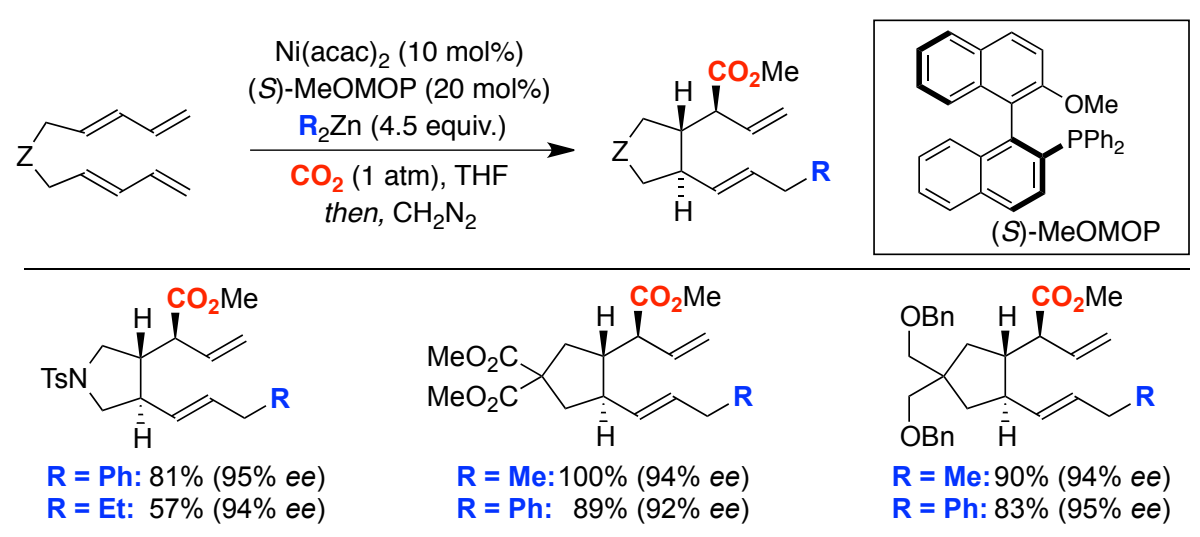

Scheme 40. Enantioselective Ni-catalyzed cyclization/carboxylation of bis(1,3-dienes) [129]

\section{Conclusions and future perspective}

The seminal stoichiometric studies reported by Hoberg in the early 1980's revealed the unique features of nickelalactones, compounds that derive from an oxidative cyclization of olefins and $\mathrm{CO}_{2}$. This seemingly trivial discovery triggered unimaginable consequences in the field of organic synthesis, allowing for designing unconventional carboxylation techniques using $\mathrm{CO}_{2}$ as coupling partner. Indeed, the recent years have witnessed a meteoric development of $\mathrm{Ni}$ - and Fe-catalyzed carboxylation protocols, providing new dogmas for promoting $\mathrm{CO}_{2}$ insertion into unsaturated hydrocarbons, building blocks of utmost synthetic and industrial relevance. Despite the impressive preparative advances realized, there are ample opportunities in this vibrant research field as daunting challenges still need to be addressed in this area of expertise: (a) the regioselectivity of the corresponding carboxylation event is typically problematic when dealing with intermolecular techniques; (b) the vast majority of catalytic carboxylation events of unsaturated hydrocarbons remain confined to the utilization of well-defined, and air-sensitive stoichiometric organometallic species; (c) catalytic carboxylation techniques of alkenes are still rather substrate-specific, and a general solution to accommodate either styrenes or less activated $\alpha$-olefins remains rather elusive; (d) the preparation of industrially-relevant acrylic acid derivatives from ethylene requires the design of an optimal 
catalytic protocol capable of operating at high turnover numbers under mild reaction conditions; (e) catalytic enantioselective carboxylation events are scarce. Taking into consideration the impressive knowledge acquired in catalytic endeavors, we are certainly optimistic that many of these challenges will be addressed in the near future, allowing for the implementation of green, efficient and practical catalytic carboxylation methods that will create new paradigms in retrosynthetic analysis to be utilized in both industrial and academic laboratories. A long-term goal will obviously deal with the opportunity of mimicking nature by designing artificial catalytic photosynthesis in which $\mathrm{CO}_{2}$ is incorporated into organic matter with visible light.

\section{Acknowledgements}

We thank ICIQ, European Research Council (ERC-277883), MINECO (CTQ2015-65496-R \& Severo Ochoa Excellence Accreditation 2014-2018, SEV-2013-0319) and Cellex Foundation for support. E. Serrano, M. van Gemmeren and F. Juliá-Hernández thank MINECO, Alexander von Humboldt Foundation and COFUND for predoctoral and postdoctoral fellowships, respectively.

\section{References}

1. Aresta M (1999) Recovery and utilisation of carbon dioxide. RUCADI, EU Report,

2. von der Assen N, Voll P, Peters M, Bardow A (2014) Life cycle assessment of CO2 capture and utilization: a tutorial review. Chem Soc Rev 43:7982-7994.

3. Aresta M, Dibenedetto A, Angelini A (2014) Catalysis for the valorization of exhaust carbon: from CO2 to chemicals, materials, and fuels. technological use of CO2. Chem Rev 114:1709-1742.

4. Assen N, Muller LJ, Steingrube A, Voll P, Bardow A (2016) Selecting CO2 sources for CO2 utilization by environmental-merit-order Curves. Environ Sci Technol 50:1093-1101.

5. Meylan FD, Moreau V, Erkman S (2015) CO2 utilization in the perspective of industrial ecology, an overview. Journal of CO2 Utilization 12:101-108.

6. Mikkelsen M, Jørgensen M, Krebs FC (2010) The teraton challenge. A review of fixation and transformation of carbon dioxide. Energy Environ Sci 3:43-81.

7. Wang S, Du G, Xi C (2016) Copper-catalyzed carboxylation reactions using carbon dioxide. Org Biomol Chem 14:3666-3676.

8. Sekine K, Yamada T (2016) Silver-catalyzed carboxylation. DOI: 10.1039/c5cs00895f.

9. Yu D, Teong S P, Zhang Y (2015) Transition metal complex catalyzed carboxylation reactions with $\mathrm{CO}_{2}$. Coord Chem Rev 293-294:279-291.

10. Guo C-X, Yu B, Ma R, He L-N (2015) Metal-promoted carboxylation of alkynes/allenes with carbon dioxide. Current Green Chemistry 2:14-25.

11. Industrial-scale preparation of low molecular weight acids is mainly carried out by carbonylation and/or oxidation of the corresponding alcohols and light olefins. See for instance: Samel U-R, Kohler 
W, Gamer A O, Keuser U, Yang S-T, Jin Y, Lin M, Wang Z (2014) Propionic acid and derivatives. Ullmann's Encyclopedia of Industrial Chemistry 1-20.

12. Correa A, Martin R (2009) Metal-catalyzed carboxylation of organometallic reagents with carbon dioxide. Angew Chem Int Ed 48:6201-6204.

13. Brill M, Lazreg F, Cazin CSJ, Nolan SP (2016) Transition metal-catalyzed carboxylation of organic substrates with carbon dioxide. Top Organomet Chem 53:225-278.

14. Correa A, Leon T, Martin R (2014) Ni-catalyzed carboxylation of C( $\left.\mathrm{sp}^{2}\right)$ - and $\mathrm{C}\left(\mathrm{sp}^{3}\right)$-O bonds with $\mathrm{CO}_{2}$. J Am Chem Soc 136:1062-1069.

15. Moragas T, Gaydou M, Martin R (2016) Ni-catalyzed carboxylation of benzylic C-N bonds with $\mathrm{CO}_{2}$. Angew Chem Int Ed 55:5053-5057.

16. Wang X, Liu Y, Martin R (2015) Ni-catalyzed divergent cyclization/carboxylation of unactivated primary and secondary alkyl halides with $\mathrm{CO}_{2}$. J Am Chem Soc 137:6476-6479.

17. Aresta $\mathrm{M}$, Dibenedetto A, Quaranta $\mathrm{E}$ (2016) Interaction of $\mathrm{CO} 2$ with $\mathrm{C}-\mathrm{C}$ multiple bonds. Reaction mechanisms in carbon dioxide conversion: doi:10.1007/978-3-662-46831-9_5.

18. Aresta M, Nobile CF, Albano VG, Forni E, Manassero M (1975) New nickel-carbon dioxide complex: synthesis, properties, and crystallographic characterization of (carbon dioxide)bis(tricyclohexylphosphine)nickel. J Chem Soc, Chem Commun 0:636-637.

19. Rintjema J, Peña Carrodeguas L, Laserna V, Sopeña S, Kleij AW (2015) Metal complexes catalyzed cyclization with CO2. Topics in Organometallic Chemistry 53:39-71.

20. Coates GW, Moore DR (2004) Discrete metal-based catalysts for the copolymerization of CO2 and epoxides: discovery, reactivity, optimization, and mechanism. Angew Chem Int Ed 43:6618-6639. 21. G. SA-A, Sivaram S (1996) Organic carbonates. Chem Rev 96:951-976.

22. Federsel C, Jackstell R, Beller M (2010) State-of-the-art catalysts for hydrogenation of carbon dioxide. Angew Chem Int Ed 49:6254-6257.

23. Wang W, Wang S, Ma X, Gong J (2011) Recent advances in catalytic hydrogenation of carbon dioxide. Chem Soc Rev 40:3703-3727.

24. Goeppert A, Czaun M, Jones JP, Surya Prakash GK, Olah GA (2014) Recycling of carbon dioxide to methanol and derived products - closing the loop. Chem Soc Rev 43:7995-8048.

25. Matthessen R, Fransaer J, Binnemans K, De Vos DE (2014) Electrocarboxylation: towards sustainable and efficient synthesis of valuable carboxylic acids. Beilstein J Org Chem 10:2484-2500.

26. Nogi K, Fujihara T, Terao J, Tsuji Y (2016) Carboxyzincation Employing Carbon Dioxide and Zinc Powder: Cobalt-Catalyzed Multicomponent Coupling Reactions with Alkynes. J Am Chem Soc 138:5547-5550.

27. Beller M, Gu X-F (2013) Transition Metal Catalyzed Carbonylation Reactions, Springer.

28. Fujihara T, Nogi K, Xu T, Terao J, Tsuji Y (2012) Nickel-catalyzed carboxylation of aryl and vinyl chlorides employing carbon dioxide. J Am Chem Soc 134:9106-9109. 
29. Liu Y, Cornella J, Martin R (2014) Ni-catalyzed carboxylation of unactivated primary alkyl bromides and sulfonates with CO2. J Am Chem Soc 136:11212-11215.

30. Inoue Y, Itoh Y, Hashimoto H (1977) Incorporation of carbon dioxide in alkyne oligomerization catalyzed by nickel(0) complexes. Formation of substituted 2-pyrones. Chem Lett:855-856.

31. Burkhart G, Hoberg H (1982) Oxanickelacyclopentene derivatives from Nickel(0), carbon dioxide, and alkynes. Angew Chem Int Ed 21:76.

32. Sakaki S, Mine K, Taguchi D, Arai T (1993) Formation of the oxanickelacyclopentene complex from nickel(0), carbon dioxide, and alkyne. An ab initio MO/SD-CI study. Bull Chem Soc Jpn 66:3289-3299.

33. Sakaki S, Mine K, Hamada T, Arai T (1995) Formation of the oxanickelacyclopentene complex from nickel(0), carbon dioxide, and alkyne. An ab initio MO/SD-CI Study. Part II. Reactivity and regioselectivity of hydroxyacetylene. . Bull Chem Soc Jpn 68:1873-1882.

34. Graham DC, Bruce MI, Metha GF, Bowie JH, Buntine MA (2008) Regioselective control of the nickel-mediated coupling of acetylene and carbon dioxide - A DFT study. J Organomet Chem 693:2703-2710.

35. Li J, Jia G, Lin Z (2008) Theoritical Studies on Coupling Reactions of Carbon Dioxide with Alkynes Mediated by Nickel(0) Complexes. Organometallics 27:3892-3900.

36. Saito S, Nakagawa S, Koizumi T, Hirayama K, Yamamoto Y (1999) Nickel-mediated regio- and chemoselective carboxylation of alkynes in the presence of carbon dioxide. J Org Chem 64:39753978.

37. Aoki M, Kaneko M, Izumi S, Ukai K, Iwasawa N (2004) Bidentate amidine ligands for nickel(0)mediated coupling of carbon dioxide with unsaturated hydrocarbons. Chem Commun:2568-2569.

38. Takimoto M, Shimizu K, Mori M (2001) Nickel-promoted alkylative or arylative carboxylation of alkynes. Org Lett 3:3345-3347.

39. Shimizu K, Takimoto M, Mori M (2003) Novel synthesis of heterocycles having a functionalized carbon center via Nickel-mediated carboxylation: total synthesis of Erythrocarine. Org Lett 5:23232325.

40. Shimizu K, Takimoto M, Sato Y, Mori M (2006) Total synthesis of ( \pm )-erythrocarine using dienyne metathesis. J Organomet Chem 691:5466-5475.

41. Saito N, Sun Z, Sato Y (2015) Nickel-promoted highly regioselective carboxylation of aryl ynol ether and its application to the synthesis of chiral beta-aryloxypropionic acid derivatives. Chem Asian J 10:1170-1176.

42. Inoue Y, Itoh Y, Hashimoto H (1978) Oligomerization of 3-hexyne by nickel(0) complexes under $\mathrm{CO}_{2}$. Incorporation of $\mathrm{CO}_{2}$ and novel cyclotrimerization. Chem Lett:633-634.

43. Inoue Y, Itoh Y, Kazama H, Hashimoto H (1980) Reaction of dialkyl-substituted alkynes with carbon dioxide catalyzed by nickel(0) complexes. Incorporation of carbon dioxide in alkyne dimers and novel cyclotrimerization of the alkynes. Bull Chem Soc Jpn 53:3329-3333. 
44. Walther D, Schönberg H, Dinjus E (1987) Aktivierung von kohlendioxid an übergangsmetallzentren: selektive cooligomerisation mit hexin durch das katalysatorsystem acetonitril/ trialkylphosphan / nickel(0) und struktur eines nickel(0)-komplexes mit side-on gebundenem acetonitril. J Org Chem 334:377-388.

45. Tsuda T, Kunisada K, Nagahama N, Morikawa S, Saegusa T (1989) Nickel(0)-catalyzed cycloaddition of ethoxyethyne with carbon dioxide to 4,5-diethoxy- $\alpha$-pyrone. Synth Commun 19:1575-1581.

46. Tsuda T, Hasegawa N, Saegusa T (1990) Nickel(0)-catalysed novel co-oligomerization of ethoxy(trimethylsilyl)ethyne with carbon dioxide to 4,6-diethoxy-3-[1-ethoxy-2,2bis(trimethylsilyl)vinyl]-5-(trimethylsilyl)-2-pyrone. J Chem Soc, Chem Commun:945-947.

47. Hoberg H, Schaefer D, Burkhart G, Krüger C, Romao MJ (1984) Nickel(0)-induzierte C-C verknüpfung zwischen kohlendioxid und alkinen sowie alkenen. J Organomet Chem 266:203-224.

48. Mori M (2007) Regio- and stereoselective synthesis of tri- and tetrasubstituted alkenes by introduction of CO2 and alkylzinc reagents into alkynes. Eur J Org Chem 2007:4981-4993.

49. Shimizu K, Takimoto M, Sato Y, Mori M (2005) Nickel-catalyzed regioselective synthesis of tetrasubstituted alkene using alkylative carboxylation of disubstituted alkyne. Org Lett 7:195-197.

50. Sato Y, Mori M, Shimizu K, Takimoto M (2006) Effective synthesis of tamoxifen using nickelcatalyzed arylative carboxylation. Synlett 2006:3182-3184.

51. Buchwald SL, Nielsen RB (1989) Selective, zirconium-mediated cross-coupling of alkynes: synthesis of isomerically pure 1,3-dienes and 1,4-diiodo 1,3-dienes. J Am Chem Soc 111:2870-2874.

52. Fujihara T, Horimoto Y, Mizoe T, Sayyed FB, Tani Y, Terao J, Sakaki S, Tsuji Y (2014) Nickelcatalyzed double carboxylation of alkynes employing carbon dioxide. Org Lett 16:4960-4963.

53. Li S, Yuan W, Ma S (2011) Highly regio- and stereoselective three-component nickel-catalyzed syn-hydrocarboxylation of alkynes with diethyl zinc and carbon dioxide. Angew Chem Int Ed $50: 2578-2582$.

54. Fujihara T, Xu T, Semba K, Terao J, Tsuji Y (2011) Copper-catalyzed hydrocarboxylation of alkynes using carbon dioxide and hydrosilanes. Angew Chem Int Ed 50:523-527.

55. Li S, Ma S (2011) Highly selective nickel-catalyzed methyl-carboxylation of homopropargylic alcohols for $\alpha$-alkylidene- $\gamma$-butyrolactones. Org Lett 13:6046-6049.

56. Li S, Ma S (2012) CO2-activation for gamma-butyrolactones and its application in the total synthesis of (+/-)-heteroplexisolide E. Chem Asian J 7:2411-2418.

57. Wang X, Nakajima M, Martin R (2015) Ni-catalyzed regioselective hydrocarboxylation of alkynes with $\mathrm{CO} 2$ by using simple alcohols as proton sources. J Am Chem Soc 137:8924-8927.

58. Trost BM, Ball ZT (2005) Addition of metalloid hydrides to alkynes: Hydrometallation with Boron, Silicon, and Tin. Synthesis 6:853-887. 
59. Fu M-C, Shang R, Cheng W-M, Fu Y (2016) Nickel-catalyzed regio- and stereoselective hydrocarboxylation of alkynes with formic acid through catalytic CO recycling. ACS Catal 6:25012505.

60. Hoberg H, Schaefer D (1982) Nickel(0)-induzierte C-C verknupfung zwischen alkenen und kohlendioxid. J Organomet Chem 236:C28-C30.

61. Hoberg H, Schaefer D (1983) Nickel(0)-induzierte C-C verknupfung zwischen kohlendioxid und etylen sowie mono- oder di-substituierten alkenen. J Organomet Chem 1983:C51-C53.

62. Hoberg H, Peres Y, Milchereit A (1986) C-C Verknupfung von alkenen mit CO2 an Nickel(0); Herstellung von zimtsaure aus styrol. J Organomet Chem 307:C38-C40.

63. Hoberg H, Ballesteros A, Sigan A, Jegat C, Barhausen D, Milchereit A (1991) Ligandgesteuerte ringkontraktion von Nickela-funfin vierringkomplexe-neuartige startsysteme fur die praparative Chemie. J Organomet Chem 407:C23-C29.

64. Greenburg ZR, Jin D, Williard PG, Bernskoetter WH (2014) Nickel promoted functionalization of $\mathrm{CO}_{2}$ to anhydrides and ketoacids. Dalton Trans 43:15990-15996.

65. Murakami M, Ishida N, Miura T (2006) Solvent and ligand partition reaction pathways in nickelmediated carboxylation of methylenecyclopropanes. Chem Comm:643-645.

66. Guo C-H, Tian L-C, Jia J, Wu H-S (2014) Theoretical study on the nickel(0)-mediated coupling of carbon dioxide and benzylidenecyclopropane: Mechanism and selectivity. Computational and Theoretical Chemistry 1044:44-54.

67. Aresta M, Quaranta E, Tommasi I (1988) Reduction of coordinated carbon dioxide to carbon monoxide via protonation by thiols and other Bronsted acids by Ni-systems: a contribution to the understanding of the mode of action of the enzyme carbon monoxide dehydrogenase. J Chem Soc, Chem Commun:450-452.

68. Aresta M, Gobetto R, Quaranta E, Tommasi I (1992) A bonding-reactivity relationship of $\mathrm{Ni}(\mathrm{PCy} 3) 2(\mathrm{CO} 2)$ : A comparative solid-state-solution nuclear magnetic resonance study $(31 \mathrm{P}, 13 \mathrm{C})$ as a diagnostic tool to determine the mode of bonding of $\mathrm{CO} 2$ to a metal center. Inorg Chem 31:42864290.

69. Papai I, Schubert G, Mayer I, Besenyei G, Aresta M (2004) Mechanistic details of Nickel(0)assisted oxidative coupling of $\mathrm{CO} 2$ with $\mathrm{C} 2 \mathrm{H} 4$. Organometallics 23:5252-5259.

70. Yang G, Schäffner B, Blug M, Hensen EJM, Pidko EA (2014) A mechanistic study of Nicatalyzed carbon dioxide coupling with ethylene towards the manufacture of acrylic acid. ChemCatChem 6:800-807.

71. Plessow PN, Schäfer A, Limbach M, Hofmann P (2014) Acrylate formation from CO2 and ethylene mediated by Nickel complexes: A theoretical study. Organometallics 33:3657-3668.

72. Hoberg H, Peres Y, Kruger C, Tsay Y-H (1987) A 1-oxa-2-nickela-5-cyclopentanone from ethene and carbon dioxide: Preparation, structure, and reactivity. Angew Chem Int Ed 26:771-773. 
73. Hoberg H, Jenni K, Angermund K, Kruger C (1987) C-C-Linkages of ethene with CO2 on an Iron(0) complex - Synthesis and crystal structure analysis of [(PEt3)2Fe(C2H4)2]. Angew Chem Int Ed 26:153-155.

74. Kirillov E, Carpentier JF, Bunel E (2015) Carboxylic acid derivatives via catalytic carboxylation of unsaturated hydrocarbons: whether the nature of a reductant may determine the mechanism of $\mathrm{CO} 2$ incorporation? Dalton Trans 44:16212-16223.

75. Lapidus AL, Pirozhkov SD, Koryakin AA (1978) Catalytic synthesis of propionic acid by carboxylation of ethylene with carbon dioxide. Izvestiya Akademii Nauk SSSR, Seriya Khimicheskaya 12:2814-2816.

76. Williams CM, Johnson JB, Rovis T (2008) Nickel-catalyzed reductive carboxylation of styrenes using CO2. J Am Chem Soc 130:14936-14937.

77. Yuan R, Lin Z (2014) Computational insight into the mechanism of Nickel-catalyzed reductive carboxylation of styrenes using CO2. Organometallics 33:7147-7156.

78. Shirakawa E, Ikeda D, Masui S, Yoshida M, Hayashi T (2012) Iron-copper cooperative catalysis in the reactions of alkyl Grignard reagents: exchange reaction with alkenes and carbometalation of alkynes. J Am Chem Soc 134:272-279.

79. Greenhalgh MD, Thomas SP (2012) Iron-catalyzed, highly regioselective synthesis of alpha-aryl carboxylic acids from styrene derivatives and CO2. J Am Chem Soc 134:11900-11903.

80. Rio I, Claver C, van Leeuwen PWNM (2001) On the mechanism of the hydroxycarbonylation of styrene with palladium systems. Eur J Inorg Chem:2719-2738.

81. Smith BRJ, Loganathan M, Shantha MS (2010) A review of the water gas shift reaction kinetics. Int J Chem React Eng 8:1542.

82. Ostapowicz TG, Schmitz M, Krystof M, Klankermayer J, Leitner W (2013) Carbon dioxide as a $\mathrm{C}(1)$ building block for the formation of carboxylic acids by formal catalytic hydrocarboxylation. Angew Chem Int Ed 52:12119-12123.

83. González-Sebastián L, Flores-Alamo M, García JJ (2012) Nickel-catalyzed reductive hydroesterification of styrenes using CO2 and MeOH. Organometallics 31:8200-8207.

84. Wu L, Liu Q, Fleischer I, Jackstell R, Beller M (2014) Ruthenium-catalysed alkoxycarbonylation of alkenes with carbon dioxide. Nat Commun 5:3091.

85. Yu B, Diao Z-F, Guo C-X, He L-N (2013) Carboxylation of olefins/alkynes with CO2 to industrially relevant acrylic acid derivatives. Journal of CO2 Utilization 1:60-68.

86. Limbach M (2015) Acrylates from alkenes and CO2, the stuff that dreams are made of. Adv. Organomet. Chem 63:175-202.

87. Graham DC, Mitchell C, Bruce MI, Metha GF, Bowie JH, Buntine MA (2007) Production of acrylic acid through Nickel-mediated coupling of ethylene and carbon dioxide-A DFT Study. Organometallics 26:6784-6792. 
88. Fischer R, Langer J, Malassa A, Walther D, Gorls H, Vaughan G (2006) A key step in the formation of acrylic acid from $\mathrm{CO} 2$ and ethylene: the transformation of a nickelalactone into a nickelacrylate complex. Chem Comm:2510-2512.

89. Bruckmeier C, Lehenmeier MW, Reichardt R, Vagin S, Rieger B (2010) Formation of methyl acrylate from $\mathrm{CO} 2$ and ethylene via methylation of nickelalactones. Organometallics 29:2199-2202.

90. Lee SY, Cokoja M, Drees M, Li Y, Mink J, Herrmann WA, Kuhn FE (2011) Transformation of nickelalactones to methyl acrylate: on the way to a catalytic conversion of carbon dioxide. ChemSusChem 4:1275-1279.

91. Lee SYT, Ghani AA, D'Elia V, Cokoja M, Herrmann WA, Basset J-M, Kühn FE (2013) Liberation of methyl acrylate from metallalactone complexes via $\mathrm{M}-\mathrm{O}$ ring opening $(\mathrm{M}=\mathrm{Ni}, \mathrm{Pd})$ with methylation agents. New J Chem 37:3512.

92. Plessow PN, Weigel L, Lindner R, Schäfer A, Rominger F, Limbach M, Hofmann P (2013) Mechanistic details of the Nickel-mediated formation of acrylates from $\mathrm{CO} 2$, ethylene and methyl iodide. Organometallics 32:3327-3338.

93. Jin D, Schmeier TJ, Williard PG, Hazari N, Bernskoetter WH (2013) Lewis acid induced $\beta$ elimination from a nickelalactone: Efforts toward acrylate production from $\mathrm{CO} 2$ and ethylene. Organometallics 32:2152-2159.

94. Guo W, Michel C, Schwiedernoch R, Wischert R, Xu X, Sautet P (2014) Formation of acrylates from ethylene and $\mathrm{CO} 2$ on Ni complexes: A mechanistic viewpoint from a hybrid DFT approach. Organometallics 33:6369-6380.

95. Jin D, Williard PG, Hazari N, Bernskoetter WH (2014) Effect of sodium cation on metallacycle beta-hydride elimination in CO2-ethylene coupling to acrylates. Chem Eur J 20:3205-3211.

96. Lejkowski ML, Lindner R, Kageyama T, Bodizs GE, Plessow PN, Muller IB, Schafer A, Rominger F, Hofmann P, Futter C, Schunk SA, Limbach M (2012) The first catalytic synthesis of an acrylate from CO2 and an alkene-a rational approach. Chem Eur J 18:14017-14025.

97. Manzini S, Huguet N, Trapp O, Paciello RA, Schaub T (2016) Synthesis of acrylates from olefins and $\mathrm{CO}_{2}$ using sodium alkoxides as bases. Catalysis Today DOI: 10.1016/j.cattod.2016.03.025.

98. Huguet N, Jevtovikj I, Gordillo A, Lejkowski ML, Lindner R, Bru M, Khalimon AY, Rominger F, Schunk SA, Hofmann P, Limbach M (2014) Nickel-catalyzed direct carboxylation of olefins with CO2: one-pot synthesis of alpha,beta-unsaturated carboxylic acid salts. Chem Eur J 20:16858-16862.

99. Jevtovikj I, Manzini S, Hanauer M, Rominger F, Schaub T (2015) Investigations on the catalytic carboxylation of olefins with $\mathrm{CO} 2$ towards alpha,beta-unsaturated carboxylic acid salts: characterization of intermediates and ligands as well as substrate effects. Dalton Trans 44:1108311094.

100. Hendriksen C, Pidko EA, Yang G, Schaffner B, Vogt D (2014) Catalytic formation of acrylate from carbon dioxide and ethene. Chem Eur J 20:12037-12040. 
101. Manzini S, Huguet N, Trapp O, Schaub T (2015) Palladium- and Nickel-catalyzed synthesis of sodium acrylate from ethylene, $\mathrm{CO} 2$, and phenolate bases: Optimization of the catalytic system for a potential process. Eur J Org Chem 2015:7122-7130.

102. Goossen LJ, Goossen K (2008) Nachhaltigkeit durch atomökonomische Synthesen. Aktuelle Wochenschau der GDCh:18.

103. Hoberg H, Oster BW (1984) Nickel(0)-induzierte C-C-verknüpfung zwischen 1,2-dienen und kohlendioxid. J Organomet Chem 266:321-326.

104. Takimoto M, Kawamura M, Mori M (2003) Nickel(0)-mediated sequential addition of carbon dioxide and aryl aldehydes into terminal allenes. Org Lett 5:2599-2601.

105. Takimoto M, Kawamura M, Mori M (2004) Nickel-mediated regio- and stereoselective carboxylation of trimethylsilylallene under an atmosphere of carbon dioxide. Synthesis:791-795.

106. Takimoto M, Kawamura M, Mori M, Sato Y (2011) Nickel-promoted carboxylation/cyclization cascade of allenyl aldehyde under an atmosphere of CO2. Synlett:1423-1426.

107. Aoki M, Izumi S, Kaneko M, Ukai K, Takaya J, Iwasawa N (2007) Ni(0)-promoted hydroxycarboxylation of 1,2-dienes by reaction with CO2 and O2. Org Lett 9:1251-1253.

108. Dérien S, Clinet J-C, Duñach E, Périchon J (1990) Coupling of allenes and carbon dioxide catalyzed by electrogenerated Nickel complexes. Synlett 2:361-364.

109. Takimoto M, Kawamura M, Mori M, Sato Y (2005) Nickel-catalyzed regio- and stereoselective double carboxylation of trimethylsilylallene under an atmosphere of carbon dioxide and its application to the synthesis of Chaetomellic acid A anhydride. Synlett:2019-2022.

110. Walther D, Dinjus E (1982) Aktivierung von Kohlendioxid an Übergangsmetallzentren; Die Metallaringschlußreaktion zwischen Kohlendioxid und 1,3-Dienen am elektronenreichen Nickel (0)Komplexrumpf. Zeitschrift für Chemie 22:228-229.

111. Walther D, Dinjus E, Seiler J, Thanh NN, Schade W, Leban I (1983) Aktivierung von CO2 an übergangsmetallzentren: Struktur and reaktivität eines C-C-kopplungsproduktes von $\mathrm{CO} 2$ und 2.3dimethylbutadien am elektronenreichen Nickel(0). Z Naturforsch B 38:835-840.

112. Hoberg H, Apotecher B (1984) $\alpha, \omega$-Disäuren aus butadien und kohlendioxid an nickel(0). J Organomet Chem 270:c15-c17.

113. Hoberg H, Schaefer D, Oster BW (1984) Diencarbonsäuren aus 1,3-dienen und CO2 durch C-Cverknüpfung an nickel(0). J Organomet Chem 266:313-320.

114. Hoberg H, Schaefer D (1983) Sorbinsäure aus piperylen und CO2 durch C-C-Verknüpfung an nickel(0). J Organomet Chem 255:C15-C17.

115. Behr A, Kanne U (1986) Nickel complex induced C-C-linkage of carbon dioxide with trienes. J Organomet Chem 317:C41-C44.

116. Hoberg H, Jenni K, Krüger C, Raabe E (1986) C-C-Kupplung von CO2 und Butadien an Eisen(o)-Komplexen - ein neuer Weg zu $\alpha, \omega$-Dicarbonsäuren. Angew Chem 98:819-820. 
117. Geyer C, Schindler S (1998) Kinetic analysis of the reaction of isoprene with carbon dioxide and a Nickel(0) complex. Organometallics 17:4400-4405.

118. Takimoto M, Mori M (2001) Cross-coupling reaction of oxo-allylnickel complex generated from 1,3-diene under an atmosphere of carbon dioxide. J Am Chem Soc 123:2895-2896.

119. Takimoto M, Mizuno T, Sato Y, Mori M (2005) Nickel-mediated carboxylative cyclization of enynes. Tetrahedron Lett 46:5173-5176.

120. Takimoto M, Mizuno T, Mori M, Sato Y (2006) Nickel-mediated cyclization of enynes under an atmosphere of carbon dioxide. Tetrahedron 62:7589-7597.

121. Mizuno T, Oonishi Y, Takimoto M, Sato Y (2011) Total synthesis of (-)-Corynantheidine by Nickel-catalyzed carboxylative cyclization of enynes. Eur J Org Chem 2011:2606-2609.

122. Hoberg H, Gross S, Milchereit A (1987) Nickel(0)-catalyzed production of a functionalized cyclopentanecarboxylic acid from 1,3-Butadiene and CO2. Angew Chem Int Ed 26:571-572.

123. Tsuda T, Morikawa S, Sumiya R, Saegusa T (1988) Nickel(0)-catalyzed cycloaddition of diynes and carbon dioxide to give bicyclic $\alpha$-pyrones. J Org Chem 53:3140-3145.

124. Tsuda T, Morikawa S, Hasegawa N, Saegusa T (1990) Nickel(0)-catalyzed cycloaddition of silyl diynes with carbon dioxide to silyl bicyclic $\alpha$-pyrones. J Org Chem 55:2978-2981.

125. Tekavec TN, Arif AM, Louie J (2004) Regioselectivity in nickel(0) catalyzed cycloadditions of carbon dioxide with diynes. Tetrahedron 60:7431-7437.

126. Louie J, Gibby JE, Farnworth MV, Tekavec TN (2002) Efficient Nickel-catalyzed [2+2+2] cycloaddition of CO2 and diynes. J Am Chem Soc 124:15188-15189.

127. Cao T, Ma S (2016) Highly Stereo- and Regioselective Hydrocarboxylation of Diynes with Carbon Dioxide. Org Lett 18:1510-1513.

128. Takimoto M, Mori M (2002) Novel catalytic CO2 incorporation reaction: Nickel-catalyzed regioand stereoselective ring-closing carboxylation of bis-1,3-dienes. J Am Chem Soc 124:10008-10009.

129. Takimoto M, Nakamura Y, Kimura K, Mori M (2004) Highly enantioselective catalytic carbon dioxide incorporation reaction: Nickel-catalyzed asymmetric carboxylative cyclization of bis-1,3dienes. J Am Chem Soc 126:5956-5957. 